\title{
Mesenchymal Stem Cells Shift Mitochondrial Dynamics and Enhance Oxidative Phosphorylation in Recipient Cells
}

\author{
Christopher Newell ${ }^{1 *}$, Rasha Sabouny ${ }^{2}$, Dustin. S. Hittel ${ }^{2}$, Timothy E. Shutt ${ }^{1,2}$, \\ Aneal Khan ${ }^{1,3}$, Matthias S. Klein ${ }^{4}$ and Jane Shearer ${ }^{2,5}$ \\ ${ }^{1}$ Department of Medical Genetics, Cumming School of Medicine, University of Calgary, Calgary, AB, Canada, ${ }^{2}$ Department \\ of Biochemistry \& Molecular Biology, Cumming School of Medicine, University of Calgary, Calgary, AB, Canada, \\ ${ }^{3}$ Departments of Pediatrics, Cumming School of Medicine, University of Calgary, Calgary, AB, Canada, ${ }^{4}$ Department of Food \\ Science and Technology, The Ohio State University, Columbus, OH, United States, ${ }^{5}$ Faculty of Kinesiology, University of \\ Calgary, Calgary, $A B$, Canada
}

Mesenchymal stem cells (MSCs) are the most commonly used cells in tissue engineering and regenerative medicine. MSCs can promote host tissue repair through several different mechanisms including donor cell engraftment, release of cell signaling factors, and the transfer of healthy organelles to the host. In the present study, we examine the specific impacts of MSCs on mitochondrial morphology and function in host tissues. Employing in vitro cell culture of inherited mitochondrial disease and an in vivo animal experimental model of low-grade inflammation (high fat feeding), we show human-derived MSCs to alter mitochondrial function. MSC co-culture with skin fibroblasts from mitochondrial disease patients rescued aberrant mitochondrial morphology from a fission state to a more fused appearance indicating an effect of MSC co-culture on host cell mitochondrial network formation. In vivo experiments confirmed mitochondrial abundance and mitochondrial oxygen consumption rates were elevated in host tissues following MSC treatment. Furthermore, microarray profiling identified 226 genes with differential expression in the liver of animals treated with MSC, with cellular signaling, and actin cytoskeleton regulation as key upregulated processes. Collectively, our data indicate that MSC therapy rescues impaired mitochondrial morphology, enhances host metabolic capacity, and induces widespread host gene shifting. These results highlight the potential of MSCs to modulate mitochondria in both inherited and pathological disease states.

Keywords: metabolism, high-fat diet, metabolic inflammation, hepatic, mitochondrial regulation Accepted: 22 October 2018 Published: 13 November 2018

Citation:

Newell C, Sabouny R, Hittel DS,

Shutt TE, Khan A, Klein MS and Shearer J (2018) Mesenchymal Stem Cells Shift Mitochondrial Dynamics and Enhance Oxidative Phosphorylation in Recipient Cells. Front. Physiol. 9:1572. doi: 10.3389/fphys.2018.01572

\section{INTRODUCTION}

Initially identified as plastic-adherent cells, mesenchymal stem cells (MSCs) are non-hematopoietic stem cells that are capable of differentiating into a multitude of cell lineages (Colter et al., 2000; Maijenburg et al., 2012). Presenting great promise for regenerative medicine and gene-based therapy, MSCs are further characterized by their capacity for self-renewal and the ability to expand as isolated cells in vitro (Hsu et al., 2016). Known to preferentially home to sites of injury and inflammation, administered MSCs have exhibited promotion of host tissue repair through donor 
cell engraftment, release of cell signaling factors, and transfer of healthy organelles including mitochondria (Shi et al., 2010; Bernardo and Fibbe, 2012; Islam et al., 2012).

At present, little is known about the impact of MSC therapy on mitochondrial function and morphology. Mitochondrial function or the ability to generate energy through OXPHOS (oxidative phosphorylation) is vital for cell homeostasis and its dysfunction has been linked to the pathogenesis of nearly all chronic diseases (Pieczenik and Neustadt, 2007). Relevant to the studies presented in this manuscript, mitochondrial dysfunction is a key contributor to the perpetuation of metabolic inflammation caused by nutrient excess. This is accomplished, in part, through the inability to utilize excess fatty acids through inhibition of enzymatic function and the generation of excessive oxidative stress (Hotamisligil, 2006; Gregor and Hotamisligil, 2011; Satapati et al., 2015). Besides OXPHOS, mitochondrial dysfunction is also present in the examination of mitochondrial morphology, the balance of mitochondrial network formation, and fission and fusion.

Excessive mitochondrial fission is linked to mitochondrial dysfunction and reductions in OXPHOS (Wai and Langer, 2016) whereas inhibition of mitochondrial fission lessens pathological phenotypes in several experimental disease models such as; myocardial infarction (Disatnik et al., 2013), cardiac arrest (Sharp et al., 2015), acute cardiorenal syndrome (Sumida et al., 2015), glaucoma (Kim et al., 2015), neuronal ischemia (Grohm et al., 2012), traumatic brain injury (Fischer et al., 2016; Wu et al., 2016), anesthesia-induced neuronal injury (Xu et al., 2016), and multiple models of neurodegeneration (Reddy, 2014). These data demonstrate that restoring the balance between fission and fusion may be a viable therapeutic approach.

To this end, we employed both in vitro cell culture and in vivo animal experimental models to study the therapeutic impact of human-derived MSCs. Initially, skin fibroblasts from patients with mitochondrial disease were co-cultured with humanderived MSCs and their mitochondrial morphology examined as a surrogate of mitochondrial dysfunction (Giedt et al., 2016). Secondly, metabolic inflammation was induced in animals following a prolonged high-fat feeding regiment before being treated with a single dose of intravenously administered humanderived MSCs for $24 \mathrm{~h}$. Previous work from our laboratory shows that mouse-derived MSCs can improve the host metabolic capacity in a model of diet induced metabolic inflammation (Nyamandi et al., 2013). Given these data, our next question was to specifically explore the impact of human-derived MSC administration on mitochondrial function and morphology in host tissues. Mitochondrial metabolism, microarray gene expression profiling and metabolomic profiling of host liver tissue were examined. The liver was chosen as our tissue of interest because of its inherent ability to attract stem cells caused by lipid accumulation, its high concentration of mitochondria, and the presentation of metabolic inflammation following prolonged high-fat feeding (Ren et al., 2012; Zhao et al., 2015). Our hypotheses were two-fold: (1) that MSC contact co-culture would alter the mitochondrial morphology of skin fibroblasts from mitochondrial disease patients, and (2) that human-derived MSC therapy would promote cell signaling within mouse liver tissue, culminating in improved mitochondrial function, altered gene expression profiles, and differences in host metabolites.

\section{MATERIALS AND METHODS}

\section{Study Approval}

This study was carried out in accordance with the recommendations of the University of Calgary Conjoint Health Research Ethics Board's recommendations with written informed consent from all subjects. Each subject gave written informed consent in accordance with the Declaration of Helsinki and the study protocol was approved by the University of Calgary's Conjoint Health Research Ethics Board (REB13-0753). This study was carried out in accordance with the recommendations of the Canadian Council on Animal Care and the University of Calgary Animal Care and Use Committee. The protocol, under the ethics report (BI11R-29), was approved by the University of Calgary Animal Care and Use Committee.

\section{Skin Fibroblast and MSC Culture}

Four patients $(2 \mathrm{M}, 2 \mathrm{~F})$ aged $46-65$ years of age were seen in the Metabolic Clinic at Alberta Children's Hospital (Calgary, AB) and investigated for mitochondrial disease (Table 1). Four controls (2M, 2F) aged 46-62 years of age were also selected from a bank of skin fibroblasts that had previously been investigated for and found not to have a diagnosis of an inborn error of metabolism or a mitochondrial disease through the Metabolic Clinic at Alberta Children's Hospital. Patient and control skin biopsy tissues were accessed from the cell bank at Alberta Children's Hospital before being passaged and expanded into T75 flasks as per the protocol established by the Biochemical Genetics Laboratory at Alberta Children's Hospital. Cellular media, comprised of minimum essential medium (MEM) with $2 \mathrm{mM}$ glutamine (Life Technologies; Burlington, ON), 10\% fetal bovine serum (FBS) (Life Technologies), $1 \mathrm{mM}$ sodium pyruvate (Life Technologies), $20 \mathrm{mM}$ uridine (Life Technologies), and $100 \mathrm{U} / \mathrm{mL}$ PenicillinStreptomycin, was replaced after 3 successive days of cell incubation. Upon reaching confluence, skin fibroblasts were detached from their respective flasks using $3 \mathrm{~mL} 0.25 \%$ trypsin/1 mM EDTA (Life Technologies) and monitored under light microscopy to confirm successful separation. Detached skin fibroblasts were then prepared for either fixation and immunofluorescent staining (see section Immunofluorescent Staining) or re-plated for co-culture experimentation (see section Fluorescent Staining and Co-Culture). Con-currently, human bone marrow-derived MSCs were provided in kind from the University of Calgary Cellular Therapy Laboratory (Dr. Nicole Prokopishyn). MSCs were cultured using the same protocol as listed above and were grown to confluency before being prepared for co-culture experimentation. All experiments were completed using skin fibroblasts and MSCs from passages 4 to 6 .

\section{Immunofluorescent Staining}

Detached skin fibroblasts were counted (MOXI Z Mini Automated Cell Counter; ORFLO Technologies, Ketchum, ID) and seeded on three autoclaved coverslips per subject at $5.0 \times$ $10^{4}$ cells per slip using a 24 well-plate. Cells were incubated at 
TABLE 1 | Subject mtDNA mutations.

\begin{tabular}{lll}
\hline ID & Age & mtDNA mutation \\
\hline C1 & $60-64$ & None \\
C2 & $60-64$ & None \\
C3 & $45-49$ & None \\
C4 & $50-54$ & None \\
P1 & $50-54$ & m.8753_16566 \\
P2 & $60-64$ & ATPase_CytB \\
P3 & $45-49$ & m.9090_16070 \\
P4 & $60-64$ & m.9928, ATPase6 \\
\hline
\end{tabular}

Patient $(P)$ and control $(C)$ diagnoses of pathogenic mtDNA mutations were confirmed by genetic sequencing. Each cohort was comprised of two males and two females. Data were provided by the Molecular Diagnostics Laboratory at the University of Alberta (Edmonton, $A B-$ courtesy of Dr. Stacey Hume).

$37^{\circ} \mathrm{C}$ overnight before being fixed with $4 \%$ paraformaldehyde in phosphate buffered saline (PBS) at $37^{\circ} \mathrm{C}$ for $15 \mathrm{~min}$. Coverslips were quenched with $\mathrm{NH}_{4} \mathrm{Cl}$ for $15 \mathrm{~min}$ and washed with PBS to minimize autofluorescence. Cells were permeabilized with $0.5 \mathrm{~mL} \quad 0.2 \%$ Triton X-100 (Sigma; Oakville, ON) in PBS for $15 \mathrm{~min}$, washed with PBS, and then blocked with $10 \%$ FBS (Life Technologies) in PBS for $30 \mathrm{~min}$. Cover slips were simultaneously incubated with primary antibodies for the outer mitochondrial membrane protein TOMM20 (FL-145, Santa Cruz; Dallas, TX) and DNA (CBL-186, EMD Millipore; Etobicoke, ON) diluted to $1: 1,000$ in 5\% FBS, 95\% PBS (Life Technologies) at $37^{\circ} \mathrm{C}$ for $1 \mathrm{~h}$. Cells were washed $3 \mathrm{x}$ with PBS and then incubated with secondary antibodies (1:5,000) at RT for $1 \mathrm{~h}$. The following secondary antibodies conjugated to fluorescent dyes were used: Alexa Fluor 488 goat anti-mouse IgG (TOMM20, Molecular Probes, Eugene, OR) and Alexa Fluor 647 goat anti-rabbit IgG (DNA, Molecular Probes). Cells were mounted with DakoCytomation fluorescent mounting medium (Agilent Technologies; Santa Clara, CA) and stored at $4^{\circ} \mathrm{C}$ until microscopic examination.

\section{Fluorescent Staining and Co-culture}

Detached skin fibroblasts were counted (MOXI Z Mini Automated Cell Counter) and seeded at $7.5 \times 10^{4}$ cells on $35 \mathrm{~mm}$ dishes with embedded, uncoated glass coverslips (MatTek; Ashland, MA) and incubated overnight. Live cells were fluorescently labeled with MitoTracker Deep Red FM (Thermo Fisher Scientific; Waltham, MA) for mitochondria and QuantiT PicoGreen dsDNA (Thermo Fisher Scientific) for nDNA and mtDNA as per the manufacturer's instructions. Separately, MSC's were cultured and fluorescently labeled with MitoTracker Red CMXRos (Thermo Fisher Scientific) for mitochondria per the manufacturer's instructions. Following $1 \mathrm{~h}$ of incubation, labeled MSCs were harvested and counted (MOXI Z Mini Automated Cell Counter) before being co-cultured with existing skin fibroblast cultures at $7.5 \times 10^{4}$ cells per $35 \mathrm{~mm}$ dish. Cocultures were imaged live after an 8 -h co-culture period, with conditions replicated for each subject.

\section{Confocal Microscopy and Mitochondrial Morphology}

Mitochondrial morphology and mtDNA nucleoid structure were analyzed by the appropriate lasers using a confocal scanning microscope (Zeiss LSM 700, Carl Zeiss Microscopy; Jena, Germany). All images were collected using a 2,048 × 2,048-pixel configuration of single confocal planes. Each image was acquired and processed using the accompanying ZEN software, version 2.3 (Carl Zeiss Microscopy). Both fixed individual and live cocultured skin fibroblast cells were manually classified into one of five mitochondrial morphology categories and one of two categories for mtDNA structure. Our classification system was developed to further describe the fission and fusion morphologies as previously described (Sabouny et al., 2017). At least 150 cells from each condition were counted from multiple independent experiments and scored by eye for morphology into the indicated classes. Morphology and mtDNA structure were evaluated by blinded investigators by comparing to a set of standard images (Figure S1).

\section{Animals and Dietary Interventions}

Male C57BL/6 mice were housed in a humidity and temperature $\left(21-22^{\circ} \mathrm{C}\right)$ controlled room with a $12 \mathrm{~h} \mathrm{light/dark} \mathrm{Zeitgeber} \mathrm{cycle}$ and free access to both food and water. At 4 weeks of age, animals were placed on a high-fat diet (HF, 60\% kcal fat; D12492, Research Diets; New Brunswick, NJ) for 20 weeks in order to elicit metabolic inflammation (Guo et al., 2009). Following dietary manipulation, animals were randomly divided into two groups: MSC therapy (HFM; $n=8$ ) or saline control (HFS; $n=8$ ).

\section{MSC Therapy}

Fluorescently labeled (GFP) human bone marrow-derived MSCs were purchased from the Center for the Preparation and Distribution of Adult Stem Cells (Institute for Regenerative Medicine, TX), an NIH/NCRR (P40 RR 17447-06) supported MSC distribution center. Delivered MSCs have been shown to successfully differentiate into adipocytes, chondrocytes, and osteocytes as well as possess the following hematopoietic markers: $\mathrm{CD} 34^{-}, \mathrm{CD} 36^{-}, \mathrm{CD} 117^{-}, \mathrm{CD} 45^{-}, \mathrm{CD} 29^{+}, \mathrm{CD} 44^{+}$, $\mathrm{CD}_{49} \mathrm{c}^{+}, \mathrm{CD}_{49 \mathrm{f}^{+}}, \mathrm{CD} 9^{+}, \mathrm{CD}^{+} 0^{+}, \mathrm{CD}_{105^{+}}$, and $\mathrm{CD} 166^{+}$ (Oh et al., 2012). Cells were passaged and expanded per the same protocol as described previously. Upon reaching confluence, MSCs were detached from their respective flasks using $3 \mathrm{~mL} \quad 0.25 \%$ trypsin/1 mM EDTA (Life Technologies) and monitored under light microscopy to establish successful separation. Detached MSC were then purified through three stages of centrifugation and counted using a hemocytometer before being suspended in PBS at a density of $7.5 \times 10^{5}$ cells per $0.2 \mathrm{ml}$. Following dietary intervention, animals were injected with equal volumes $(0.2 \mathrm{~mL})$ of $7.5 \times 10^{5} \mathrm{MSCs}$ or PBS via the tail vein. All experiments were completed using MSCs from a single donor and passages 4-6. Twenty-four hours post-injection the animals were weighed and then sacrificed via cervical dislocation. Tissues (liver and whole blood) were removed and either kept fresh or flash frozen and stored at $-80^{\circ} \mathrm{C}$ for future testing. 


\section{Sequence-Specific Qualitative PCR}

Total genomic DNA was extracted from $25 \mathrm{mg}$ of frozen liver tissue using the QIAamp DNA Mini Kit (Qiagen; Germantown, MD). DNA concentrations were measured using a NanoDrop-1,000 (Thermo Fisher Scientific). Distinct human and mouse-specific primer pairs were used to distinguish effective administration of MSCs, as documented previously (Alcoser et al., 2011), with primers synthesized by the University of Calgary Core DNA Services (Table S1). A positive human control was obtained from a cultured fibroblast cell line. PCR was performed using undiluted genomic DNA (150-200 ng) on a Mastercycler ep Gradient S (Eppendorf; Hamburg, Germany). PCR conditions were as follows: $95^{\circ} \mathrm{C}-5 \mathrm{~min}, 30$ cycles of $\left(94^{\circ} \mathrm{C}-45 \mathrm{~s}, 60^{\circ} \mathrm{C}-30 \mathrm{~s}, 72^{\circ} \mathrm{C}-90 \mathrm{~s}\right), 72^{\circ} \mathrm{C}-10 \mathrm{~min}$. Amplified PCR products were run undiluted on a $2 \%$ agarose gel plus ethidium bromide $(0.5 \mu \mathrm{g} / \mathrm{ml})$.

\section{Mitochondrial Respirometry}

Fresh liver tissue from the upper left lobe was rinsed and homogenized in liver mitochondria isolation buffer $(250 \mathrm{mM}$ sucrose, $2 \mathrm{mM} \mathrm{KH}_{2} \mathrm{PO}_{4}, 1 \mathrm{mM}$ EGTA, $20 \mathrm{mM}$ Tris-HCl, pH 7.4 at $4{ }^{\circ} \mathrm{C}$ ) (Sigma) before undergoing differential centrifugation, according to previous procedures with slight variations (Frezza et al., 2007). Mitochondrial protein quantification was performed with bovine serum albumin as the standard using the Bradford method (Bio-Rad). High-resolution respirometry measurements were then performed on isolated mitochondria samples using the Oroboros Oxygraph-2k (Oroboros Instruments; Innsbruck, Austria) at $37^{\circ} \mathrm{C}$ in MiR05 (0.5 mM EGTA, $3 \mathrm{mM}$

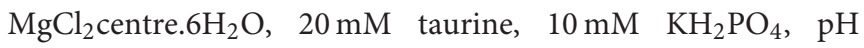
7.1) (Sigma). Each sample was run in duplicates to ensure repeatability of results. Substrate additions were as follows (final concentration): $10 \mathrm{mM}$ glutamate plus $0.5 \mathrm{mM}$ malate, $2 \mathrm{mM}$ ADP, and $2 \mu \mathrm{g} / \mathrm{ml}$ oligomycin (Sigma). A final addition of $10 \mathrm{mM}$ cytochrome $\mathrm{c}$ was added to establish the intactness of the mitochondrial outer membrane (Figure S2).

\section{Mitochondrial Enzyme Activities}

Citrate synthase activity, a mitochondrial enzyme and marker of mitochondrial content, was measured from liver homogenates using a spectrophotometric method (Tweedie et al., 2011). Total liver superoxide dismutase (SOD) activity was assessed as previously described, with slight variations (Gianni et al., 2004). Liver homogenate protein quantification was performed with bovine serum albumin as the standard using the Bradford method (Bio-Rad; Des Plaines, IL). Procedures were performed on a DU 800 Spectrophotometer (Beckman Coulter; Mississauga, $\mathrm{ON})$ with data normalized to $\mathrm{mg}$ protein.

\section{Liver Mitochondrial ROS Production}

Fresh liver tissue from the upper right lobe was rinsed, homogenized and centrifuged in order to isolate hepatic mitochondria (Frezza et al., 2007). Mitochondrial protein quantification was performed with bovine serum albumin as the standard using the Bradford method (Bio-Rad). Liver mitochondrial reactive oxygen species (ROS) production was measured using fluorometry (Hitachi F-2700 Spectrophotometer,
Hitachi America; Elk Grove Village, IL) according to previous research (Nyamandi et al., 2013).

\section{Microarray Gene Expression Profiling}

Expression profiling was performed as described previously (Hittel et al., 2003), using $5 \mu \mathrm{g}$ of total RNA extracted from frozen liver tissue from animals in both groups $(n=7$ per group due to damage of one sample per group in transit) (Hittel et al., 2010). Biotinylated cRNA was synthesized from cDNA and fragmentation as detailed in the manufacturer's protocol for GeneChip Mouse Gene 1.0 ST microarrays (Affymetrix; Santa Clara, CA). Stringent quality control methods were employed (Hittel et al., 2005). Each array fulfilled the following quality control measures: average cRNA fold changes of 15.6, average scaling factor of 3.9 to a target intensity of 500, average "present" calls $43 \%$, showed $>80 \%$ present calls, consistent values across samples, average $5^{\prime} / 3^{\prime}$ ratios of housekeeping genes, and internal probe set controls was 0.81 (Hubal et al., 2011). All microarray data are to be published and freely accessible at NCBI-GEO (GSE121879).

\section{RNA Extraction and qRT-PCR}

Total RNA was extracted from $25 \mathrm{mg}$ of frozen liver tissue using the PureLink RNA Mini Kit (Life Technologies) and was measured using a NanoDrop-1000 (Thermo Fisher Scientific). Reverse transcription was performed with $1 \mu \mathrm{g}$ of RNA using the iScript cDNA Synthesis Kit (Bio-Rad). cDNA products were quantified using a NanoDrop-1000 (Thermo Fisher Scientific). qRT-PCR was performed in $20 \mu \mathrm{l}$ reaction volumes using $50 \mathrm{ng}$ of cDNA. Representative genes, 3 upregulated and 3 downregulated, were randomly selected for measurement using qRT-PCR to verify DNA microarray results. qRT-PCR primers were synthesized by the University of Calgary Core DNA Services (Table 1). The qRT-PCR conditions were as follows: $95^{\circ} \mathrm{C}-2 \mathrm{~min}, 40$ cycles of $\left(95^{\circ} \mathrm{C}-30 \mathrm{~s}, 60^{\circ} \mathrm{C}-30 \mathrm{~s}, 72^{\circ} \mathrm{C}-30 \mathrm{~s}\right)$, $72^{\circ} \mathrm{C}-2 \mathrm{~min}$. Samples were run in triplicate on the same reaction plate using the CFX96 Real-Time PCR Detection System (BioRad) with $\beta$-actin as a loading control. Data analysis was performed in accordance with previously published work using the $2^{-\Delta \Delta C t}$ method (Lee et al., 2012).

\section{Metabolomics Sample Preparation}

Liver samples were prepared according to previously published protocols (Wu et al., 2008). Briefly, frozen samples of roughly $100 \mathrm{mg}$ were homogenized in a Fastprep-24 homogenizer (MP Biomedicals; Santa Ana, CA) using $1.4 \mathrm{~mm}$ ceramic beads ("Lysing Matrix D," MP Biomedicals). Before homogenization, $400 \mu \mathrm{L}$ cold methanol and $85 \mu \mathrm{L}$ water were added. After homogenization, $400 \mu \mathrm{L}$ chloroform and $200 \mu \mathrm{L}$ water were added and the samples kept on ice for $10 \mathrm{~min}$. Samples were centrifuged for $5 \mathrm{~min}$ at $2,000 \times \mathrm{g}$ at $4^{\circ}$. The aqueous and the lipophilic layer were collected separately and evaporated in a Vacufuge Concentrator 5301 (Eppendorf). Evaporated aqueous extracts were dissolved in $400 \mu \mathrm{L}$ water and mixed with $200 \mu \mathrm{L}$ buffer containing TSP and $50 \mu \mathrm{L}$ deuterium oxide. Evaporated lipophilic extracts were dissolved in deuterated 
chloroform containing octamethylcyclotetrasiloxane (OMS) as internal standard (Thomas et al., 2012).

\section{Metabolomics NMR Measurements}

$1 \mathrm{D}{ }^{1} \mathrm{H}$ NOESY spectra were measured at $298 \mathrm{~K}$ on an Avance II $600 \mathrm{MHz}$ NMR spectrometer equipped with a triple-resonance probe, z-gradients, and an automatic sample changer (Bruker BioSpin; Milton, ON). Spectra of aqueous liver extracts were collected using water presaturation and spoil gradients for water suppression. Acquisition and processing parameters were chosen as published before (Gronwald et al., 2008).

\section{Metabolomics Data Preparation}

NMR spectra of aqueous extracts were split into equally sized bins of $0.01 \mathrm{ppm}$ width in the range of 0.5-9.5 ppm using AMIX version 3.9.15 (Bruker BioSpin). Regions containing water and methanol were excluded from binning. Bin intensities were scaled to the TSP signal and then corrected for sample weight. Bins with a mean intensity of $<3.5$ times the noise level were excluded from further analysis. Lipid signals were integrated from the lipophilic liver extracts relative to the OMS peak and then corrected for sample weight (Klein et al., 2010a). Signals corresponding to saturated, unsaturated, and polyunsaturated fatty acid bonds, as well as total cholesterol were integrated for further analysis. Total lipids, Saturation Index (SI), Unsaturation Index (UI), Polyunsaturation Index (PUI), and Polyunsaturated Fatty Acids/Monounsaturated Fatty Acids (PUFA/MUFA) were calculated as described before (Klein et al., 2010b).

\section{Statistical Analyses}

Statistical analysis for mitochondrial morphology, animal characteristics, respirometry, mitochondrial enzyme activity, and ROS production was performed using GraphPad Prism for Windows, Version 7.01 (GraphPad Software Inc.; La Jolla, CA). Differences between treatment groups were determined by Student's unpaired, two-tailed $t$-tests where $p<0.05$ was significant. Data are expressed as mean \pm SEM. For microarray data, expression values for probe sets were generated from the probe logarithmic intensity error (PLIER) algorithm in Expression Console (Affymetrix) and imported directly into the Partek Genomics Suite, version 6.2 (Partek; St. Louis, MO) for statistical processing. Microarray metadata complied with MIAME standards, and all samples will be submitted to the NCBI GEO database for public access. All outputs were filtered with a $p<0.01$ cut-off. Following these analyses, a Bonferroni multiple testing correction was applied to reduce the false positive rate. Apart from data sets uploaded into pathway generation software, a 1.2-fold change filter was also applied. Lists of probe sets passing the $p$-value filter for differential expression between genotype groups were imported into Ingenuity Pathway Analysis software, version 7.5 (Ingenuity Systems; Redwood City, CA), for network and pathway generation. In order to examine common pathways, the Database for Annotation, Visualization and Integrated Discovery (DAVID) was employed (Huang et al., 2009a,b). All statistical analyses of metabolite data were performed in
$R$ version 3.2.3. Metabolic differences were assessed using Mann-Whitney U-tests including False Discovery Rate (FDR) controlling. Significance was assumed for FDR values below $20 \%$.

\section{RESULTS}

\section{Co-culture of Patient Skin Fibroblasts With MSC Rescues Aberrant Mitochondrial Morphology}

To examine the effect of MSCs using an in vitro model of mitochondrial dysfunction (fission morphology), skin fibroblasts from patients with long-standing, well-characterized mitochondrial disease, and matched healthy controls underwent contact co-culture with MSCs. As opposed to traditional measures of mitochondrial function (i.e., OXPHOS), mitochondrial morphology was studied since each cell population could be separately identified following staining and visualization in real-time. Mitochondrial morphology was classified into one of five categories ranging from fragmented to hyper-fused and mitochondrial DNA (mtDNA) structure into normal or clustered categories (Figure S1). The mitochondrial morphology of patient cells was significantly more fragmented than healthy controls, demonstrating a fission morphology (Figures 1, 2; $p<0.05$ ). Fission morphology has been welldocumented in models of mitochondrial disease and is hypothesized to influence human disease pathologies through mediation of: low ATP production, low-grade inflammation, and mitochondrial network fragmentation (Archer, 2013). It is also consistent with previous cell culture knockdown models where excessive fission induces severe cellular defects and decreased ATP levels (Jheng et al., 2012). When comparing mtDNA structure there was a higher prevalence of mtDNA clusters in patient cells (Figure 3; $p<0.05$ ). The aggregation of mtDNA into nucleoid clusters has been studied as a physiologic response to mtDNA stressors (Alán et al., 2016). Following MSC contact co-culture there was a rescue of the initial fission morphology, shifting patient mitochondria to an elongated fusion pattern (Figure 2; $p<0.05$ ). Furthermore, there was a $34 \%$ decrease in the percent of patient cells with clustered mtDNA nucleoids following MSC co-culture (Figure 3; $p<$ 0.05). Live cell imaging was also performed to capture transfer of labeled mitochondrial components from MSCs to fibroblasts. As identified in previously published work, bi-directional transfer of labeled mitochondrial components was identified (Shi et al., 2010; Bernardo and Fibbe, 2012; Islam et al., 2012) (Video S1). Together, these results indicate MSCs induce morphologic shifts in a cell culture model of mitochondrial dysfunction, and that this may be a result of bi-directional mitochondrial transfer.

\section{Mitochondrial Function Is Improved Following MSC Therapy}

In the next set of experiments, we sought to examine mitochondrial function with MSC administration in a model of impaired mitochondrial respiration, as opposed to overt mitochondrial disease resulting from genetic mutation. To 


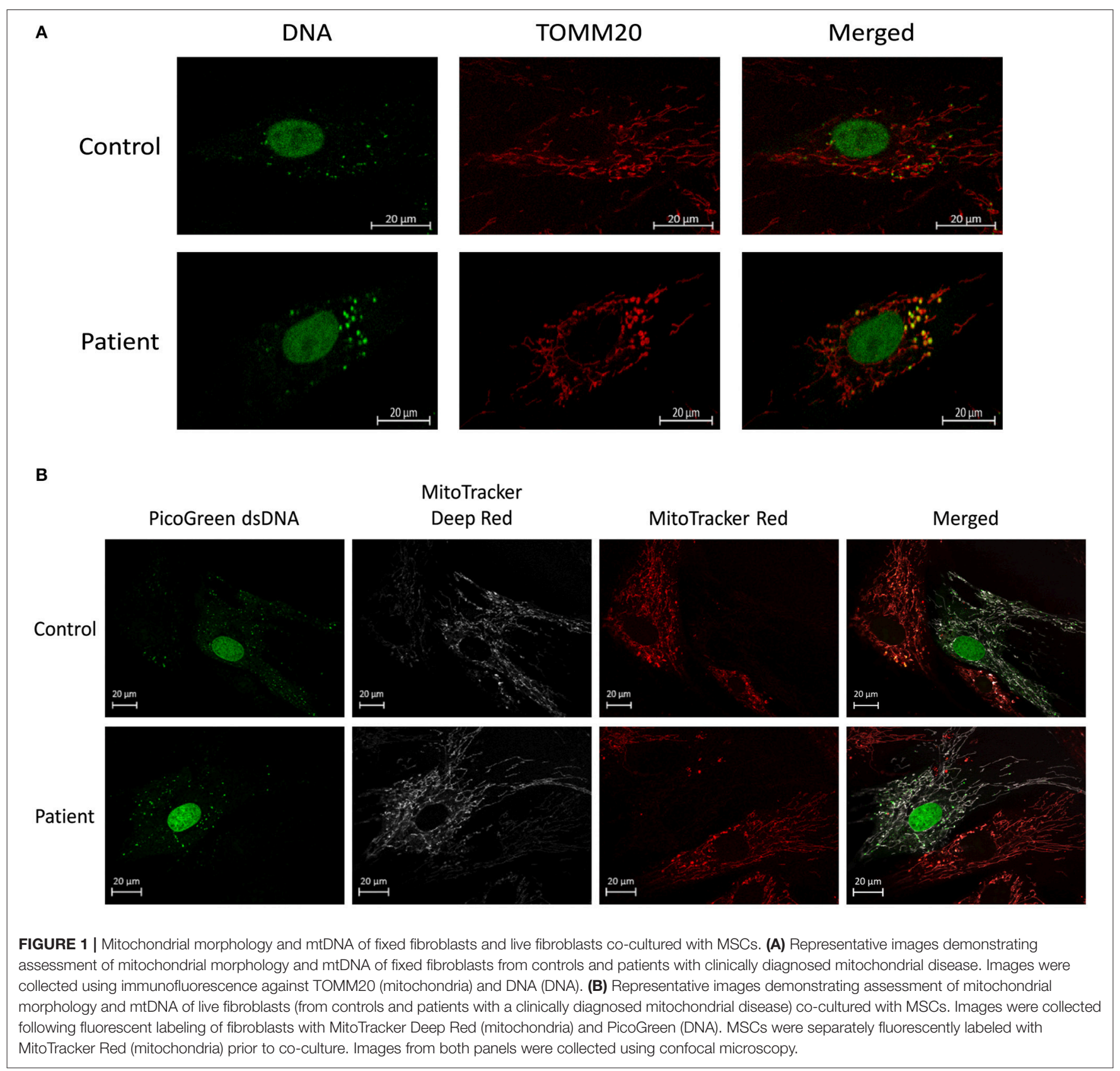

this end, we chose a sub-pathological state of mitochondrial dysfunction by exposing animals to prolonged high-fat feeding (20 week), a well-characterized model known to perturb mitochondrial function (Anderson et al., 2009; Nyamandi et al., 2013). MSC were intravenously administered via the tail vein for a $24 \mathrm{~h}$ period prior to sacrifice.

To confirm MSC presence following transplantation, qualitative PCR was used to detect sequence-specific genomic DNA in the liver. This method was employed due to complications in distinguishing fluorescently labeled MSCs from autofluorescence of liver tissue during confocal imaging following quenching of tissue. Appropriately, PCR primers specific to the mouse sequence of prostaglandin $\mathrm{E}$ receptor 2 (PTGER2) confirmed the presence of genomic mouse DNA in both high-fat saline treated (HFS) and high-fat MSC treated (HFM) groups (Figure 4A). Human specific PCR primers for a non-homologous region of the PTGER2 gene (Alcoser et al., 2011) were only successful in amplifying genetic material from HFM samples, providing evidence that MSC were only present within the liver tissue of MSC treated animals (Figure 4B).

To examine mitochondrial function with MSC treatment, specific mitochondrial complex activity was assessed by highresolution respirometry. Evaluation of respirometry data, for both basal oxygen consumption supported by glutamate and 


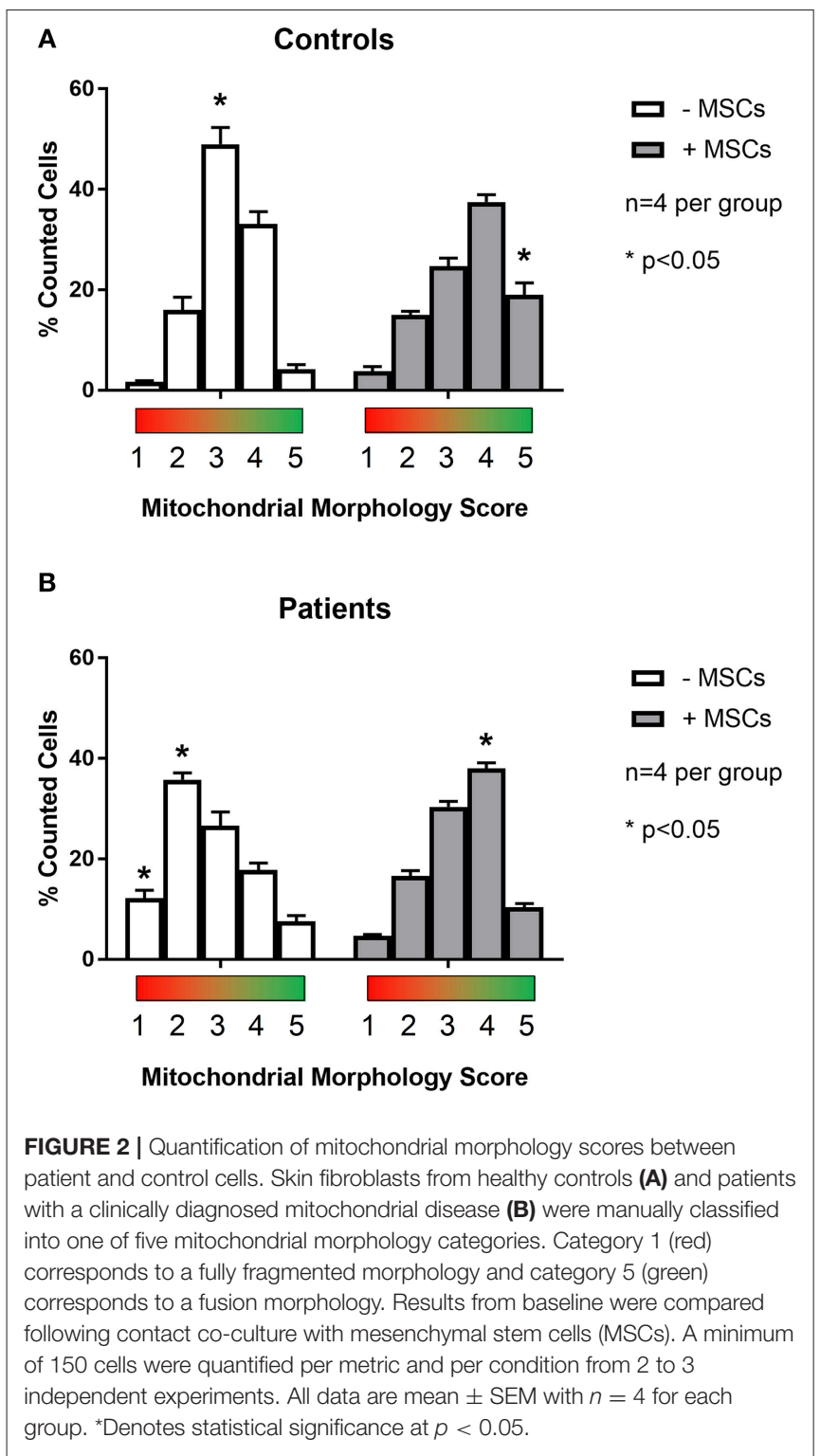

malate, oligomycin mediated proton leak (a state 4 condition) and the respiratory control ratio (RCR; State 3/State 4), a measure of mitochondrial oxidative coupling efficiency, was similar between groups (Table 2; $p<0.05$ ). However, maximal complex I oxygen consumption as assessed by the addition of ADP (a state 3 condition), was higher in HFM animals by $46 \%$ $(22.4 \pm 1.0$ vs. $32.8 \pm 3.8, p<0.05)$. Next, we sought to explain these alterations in oxidative metabolism by measuring citrate synthase enzyme activity, a marker of mitochondrial abundance. High fat fed animals treated with MSCs demonstrated a 1.5-fold increase in citrate synthase enzyme activity compared to controls, indicating a mechanism responsible for the oxidative changes (15.5 \pm 1.0 vs. $23.7 \pm 1.1, \mu \mathrm{m} / \mathrm{min} / \mathrm{mg}$ protein liver $p<0.05)$. Taken together, these findings indicate that MSC administration increases both maximal oxidative capacity and mitochondrial abundance.

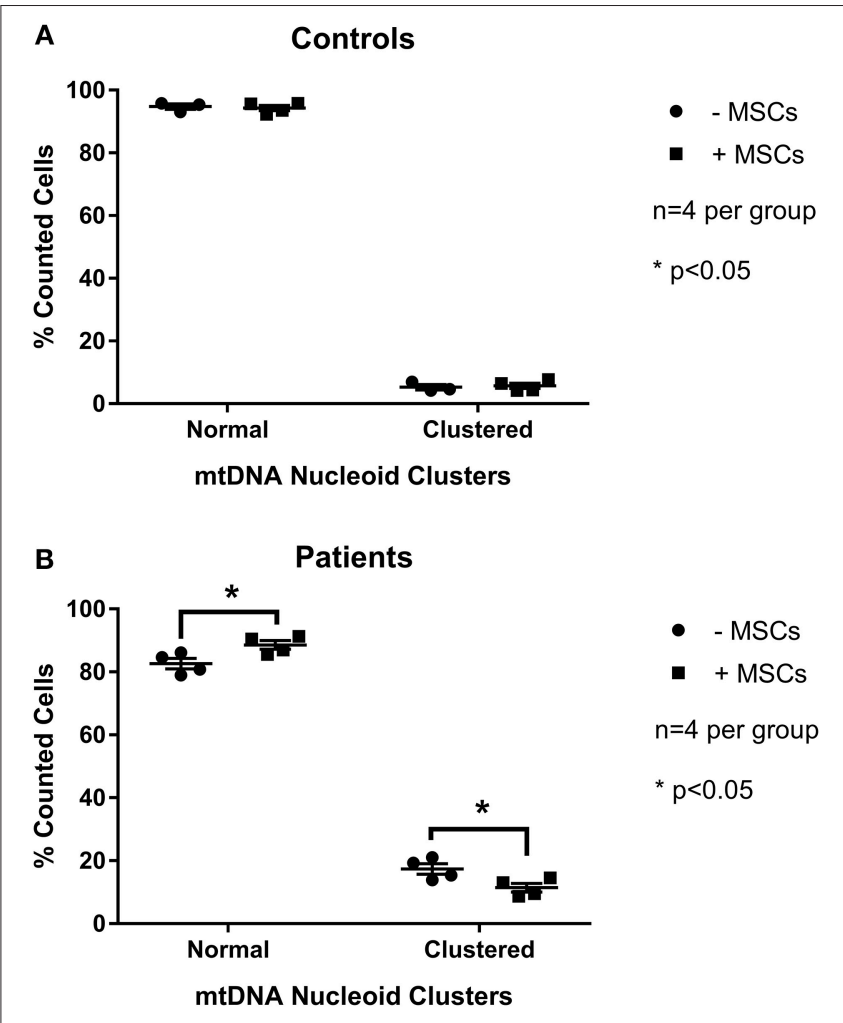

FIGURE 3 | Quantification of mitochondrial mtDNA nucleoid clusters between patient and control cells. Skin fibroblasts from healthy controls (A) and patients with a clinically diagnosed mitochondrial disease (B) were manually classified into one of twocategories for mtDNA structure. Results from baseline were compared following contact co-culture with mesenchymal stem cells (MSCs). A minimum of 150 cells were quantified per metric and per condition from 2 to 3 independent experiments. All data are mean \pm SEM with $n=4$ for each group. *Denotes statistical significance at $p<0.05$.

\section{Enhanced ROS Production at Complex III With MSC Administration}

As a metabolic by-product to mitochondrial metabolism, ROS have been implicated as both a molecule involved in healthy cell signaling, and as a damaging source of oxygen free radicals responsible for oxidative stress and mitochondrial dysfunction (Zorov et al., 2014). To assess the changes associated with mitochondrial abundance and oxidative capacity, we examined levels of oxidative stress by measuring $\mathrm{H}_{2} \mathrm{O}_{2}$ generation from liver mitochondrial isolates (Figure 5A). Neither complex I mediated respiration (a state 3 condition) in the presence of ADP or rotenone inhibition caused altered $\mathrm{H}_{2} \mathrm{O}_{2}$ production between groups. However, adding complex III inhibitor and mitochondrial membrane depolarization agent antimycin A caused increased ROS production in HFM animals $(p<$ 0.05) indicating that MSC treatment elevated mitochondrial membrane potential (Rego et al., 2001). To determine if MSC therapy simply increased the capacity of the liver to sequester excess superoxide anion production, we also studied SOD enzyme activity (Figure 5B). No differences were noted between total, cytosolic $(\mathrm{Cu} / \mathrm{Zn})$, or mitochondrial 


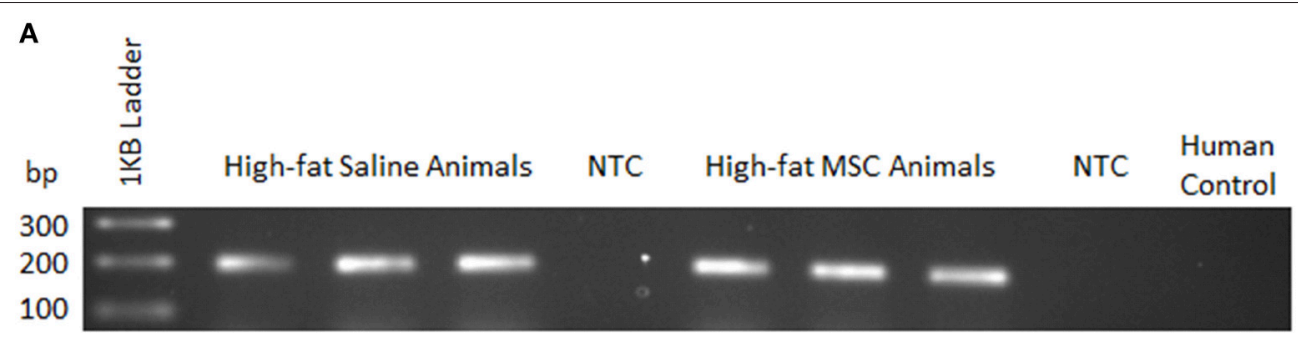

B

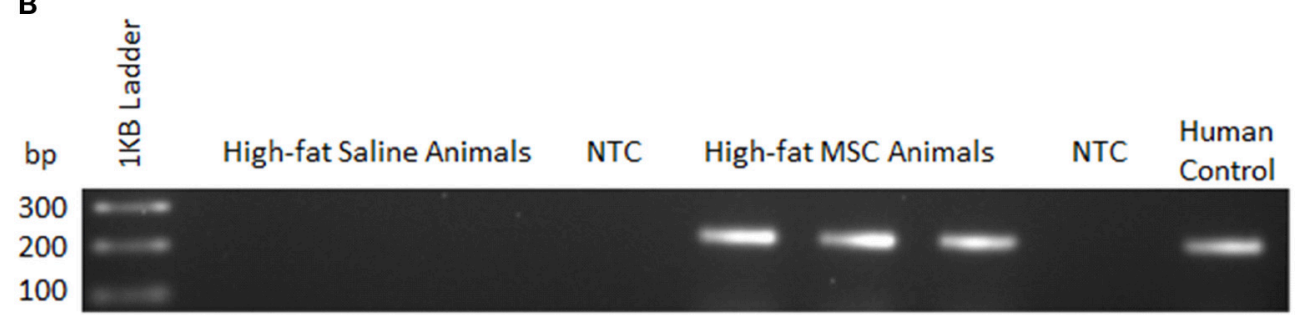

FIGURE 4 | MSC detection using sequence-specific qualitative PCR. Liver tissue homogenates were used to detect mouse and human genomic DNA $24 \mathrm{~h}$ following control (saline) or MSC therapy into C57BL/6 mice. (A) Mouse-forward and common reverse PCR primers for prostaglandin E receptor 2 (PTGER2) were amplified to detect the presence of mouse-specific genetic material. (B) Human-forward and common reverse PCR primers for a non-homologous region of PTGER2 were amplified to detect the presence of human-specific genetic material. Human control samples were isolated from a cultured human cell line. NTC, no template control.

TABLE 2 | Mitochondrial respirometry performed on mitochondria isolated from liver tissue homogenates.

\begin{tabular}{lrr}
\hline & \multicolumn{2}{c}{ High-fat } \\
\cline { 2 - 3 } & \multicolumn{1}{c}{ Saline } & MSC \\
\hline Glutamate + Malate & $2.4 \pm 0.1$ & $3.0 \pm 0.5$ \\
ADP & $22.4 \pm 1.0$ & $32.8 \pm 3.8^{*}$ \\
Oligomycin & $2.6 \pm 0.1$ & $3.4 \pm 0.5$ \\
RCR & $9.4 \pm 0.6$ & $10.0 \pm 1.0$ \\
Citrate synthase activity & $15.5 \pm 1.0$ & $23.7 \pm 1.1^{*}$
\end{tabular}

Basal oxygen consumption supported by glutamate and malate through complex I (Glutamate + Malate), maximal complex I oxygen consumption (ADP), oligomycin mediated proton leak (Oligomycin), and respiratory control ratio (RCR), a measure of mitochondrial oxidative coupling efficiency. Respirometric data was normalized to $\mathrm{mg}$ of mitochondrial protein. Data are mean \pm SEM with $n=8$ for both groups. *Denotes statistical significance at $p<0.05$.

(Mn) SOD enzyme activity. The evidence of heightened ROS signaling and improved mitochondrial function suggests that healthy cell signaling regulation may be restored by MSC exposure.

\section{Widespread Hepatic Gene Shifting Following MSC Therapy}

To examine the effect of MSC therapy on global gene expression and cell signaling pathways, microarray gene expression analysis was performed on liver tissues. Results identified 226 genes with significant differential expression between groups $(p<0.01)$. Verification of microarray results was performed on selected genes using qRT-PCR from both upregulated and downregulated categories (Table 3 ). The direction and magnitude of fold change was consistent between both microarray and qRT-PCR results.

Networks of closely related genes were also analyzed for differences following MSC therapy. These were then classified using common variations among genes utilizing functional categories derived from enriched gene ontology terms. Each of the five most variable categories contained a collective group of genes that were significantly upregulated in HFM liver samples (Figure 6; $p<0.001$ for each category). These functional categories were further stratified as to describe their physiological function (Table 4). Cell-to-cell signaling and interaction was one gene network significantly elevated following MSC therapy $(p<0.05)$. Coinciding with our ROS data, these results show that cell signaling may be reinvigorated with MSC treatment through elevated ROS production.

To identify common pathways impacted by MSC therapy, data were also analyzed using the Kyoto Encyclopedia of Genes and Genomes Pathway Database (Kanehisa et al., 2016). Using this tool, functional annotation was performed to identify primary up and down activated pathways. Analysis revealed actin cytoskeleton regulation as the primary pathway activated by MSC administration $(p<0.001)$, and retinol metabolism as the primary deactivated pathway $(p<0.001)$. Relevant to actin cytoskeleton regulation, organelle transfer via tunneling nanotubes with MSC treatment has been previously described (Islam et al., 2012). Since actin cytoskeleton proteins are known to directly interact with mitochondria for migratory purposes (Stephen et al., 2015), elevated activity of this pathway may be related to the movement of healthy MSC donor mitochondria to sites of mitochondrial impairment and metabolic stress (Anesti and Scorrano, 2006). 


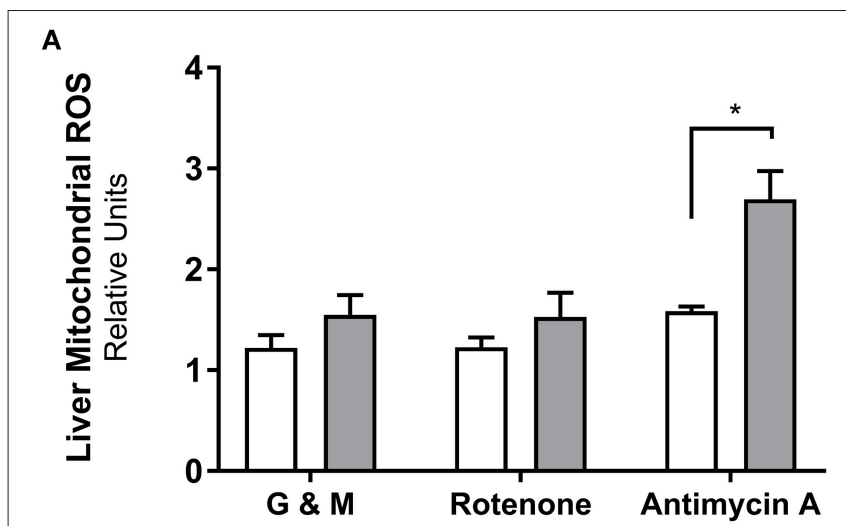

B

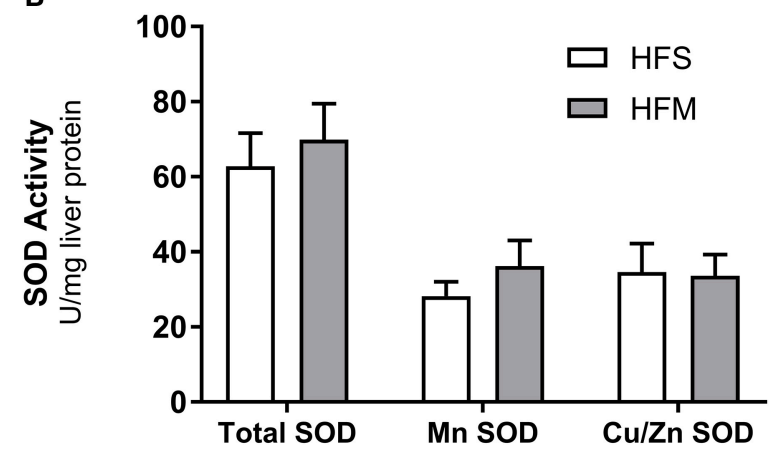

FIGURE 5 | Detection of reactive oxygen species production and superoxide dismutase enzyme activity from liver homogenates. Following control (saline) or MSC therapy, liver homogenates were used to quantify the generation of reactive oxygen species (ROS) and the free radical scavenging enzyme superoxide dismutase (SOD). (A) Relative rates of $\mathrm{H}_{2} \mathrm{O}_{2}$ production as a function of ROS generation. Mitochondria isolated from liver tissue were stimulated in the presence of ADP under a variety of conditions including: glutamate and malate as substrates ( $G$ \& $M$; complex I), rotenone as an inhibitor (complex I), and antimycin as an inhibitor (complex III). (B) SOD enzyme activity measured from liver homogenates. SOD activity is stratified into manganese ( $\mathrm{Mn}$ ) or zinc and copper ( $\mathrm{Zn}$ and $\mathrm{Cu}$ ) fractions, with total representing both portions combined. SOD data are normalized to $\mathrm{mg}$ of liver protein and ROS data are normalized to $\mathrm{mg}$ of mitochondrial protein. All data are mean \pm SEM with $n=8$ for both groups. ${ }^{*}$ Denotes statistical significance at $p<0.05$.

\section{Liver Fatty Acid Profile Is Differentially Affected by MSC Therapy}

To understand how MSC induced alterations in mitochondrial morphology and function may be impacting metabolism, tissue specific metabolomics analyses were performed by proton nuclear magnetic resonance spectroscopy. Both aqueous and non-aqueous fractions were examined. No alterations between HFM or HFS were observed for any TCA intermediates or other aqueous metabolites $(p>$ 0.05). However, an increase in lipid saturation and decrease in lipid unsaturation were identified in the HFM group $(p<0.05)$, while total lipid content remained constant (Figure 7).

\section{DISCUSSION}

Over the past decade the number of clinical trials involving MSCs has steadily increased, resulting in MSCs being the most commonly used cell type in tissue engineering and regenerative medicine (Wei et al., 2013). However, the nature and function of MSCs remains unclear, often impeding the effectiveness in treating various diseases (Squillaro et al., 2016). As a complex and multi-factorial disease phenotype, altered mitochondrial morphology and respiration is a feature of many chronic diseases, including those involving the heart (Rosca and Hoppel, 2013), skeletal muscle (Báez et al., 2015), liver (Lane et al., 2016), and brain (Navarro and Boveris, 2010). We therefore set out to examine both in vitro cell culture and in vivo animal experimental models to study the therapeutic impact of MSC treatment on mitochondrial function and dynamics. Major findings of this study are as follows: (1) MSC contact co-culture rescues aberrant mitochondrial morphology in skin fibroblasts from patients diagnosed with pathogenic mtDNA mutations; (2) in the context of a high fat diet, MSCs successfully xenograft into mouse liver; (3) MSC therapy increases both metabolic capacity and ROS production of the host liver; and (4) MSC therapy results in widespread host gene shifting as well as metabolomic alterations to lipid saturation within the liver.

As highly dynamic organelles, mitochondria undergo constant flux between fission and fusion morphology. Collectively termed mitochondrial dynamics, both fission and fusion structures have been tightly linked to mitochondrial function and metabolism (Liesa et al., 2009). Excessive fission has been associated with decreased membrane potential, ATP synthesis, oxygen consumption, and increased proton leak, while excessive fusion induces the opposite phenotype (elevated membrane potential, ATP synthesis, oxygen consumption, and decreased proton leak) (Sebastián et al., 2017). Known to confer metabolic benefits through the transfer of healthy mitochondria to damaged tissues (Ahmad et al., 2014), the role of MSCs to impact mitochondrial dynamics of the host mitochondrial pool is unknown. To this end, we performed a contact co-culture with MSCs comparing skin fibroblasts from healthy controls to mitochondrial disease patients (Figures 1, 2). The baseline fragmentation phenotype in patient fibroblasts was rescued by co-culture with MSCs, shifting the mitochondrial morphology to resemble control cells. Associated with improved metabolic output, shifting to an elongation morphology suggests an improvement to existing mitochondrial dysfunction. Live cell imaging was also performed and captured the presence of labeled mitochondrial components transiting from MSCs to fibroblasts (Video S1). The previously identified phenomenon of mitochondrial transfer may provide evidence of how MSCs alter fibroblast mitochondrial dynamics (Islam et al., 2012). On the other hand, mitochondrial function (i.e., OXPHOS) was not measured directly since contact co-culturing of MSCs and fibroblasts resulted in cell populations being difficult to separate, although future research should investigate the possibility of paracrine actions between neighboring cells. Finally, patient cells also exhibited a decrease in mtDNA aggregation after MSC co-culture, a trait linked to mtDNA stress (Alán et al., 2016). To further evaluate the capacity 
TABLE 3 | Verification of microarray using qRT-PCR on genes randomly selected from both upregulated/downregulated categories.

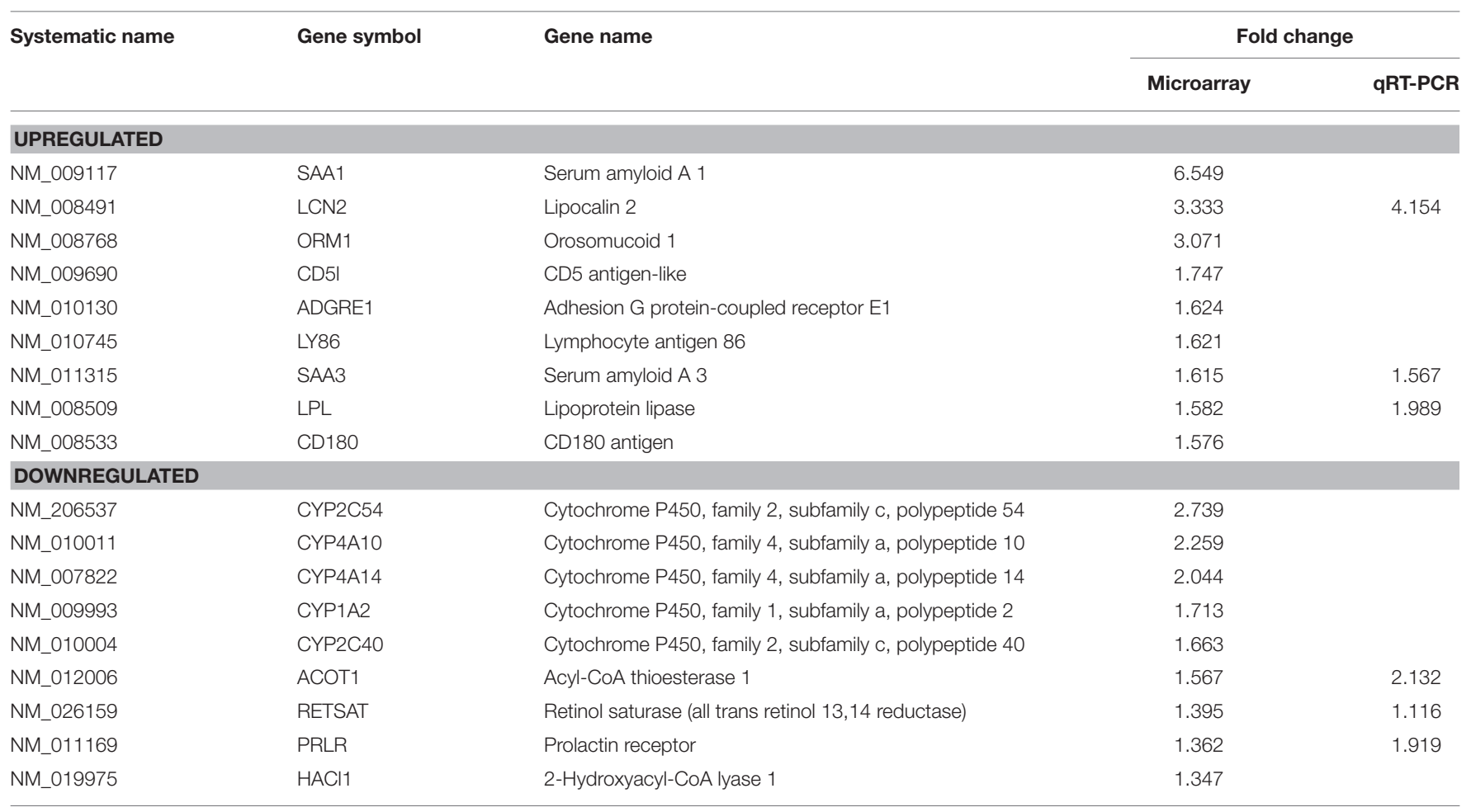

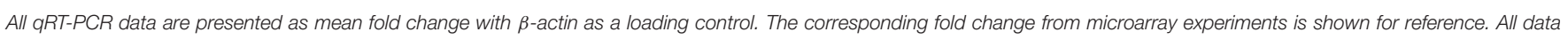
represent HFM vs. HFS ( $n=7$ for both groups).

of MSCs to impact host mitochondrial function, an in vivo animal model was studied.

Previously, our lab demonstrated that allogeneic administration of mouse-derived MSCs migrate to multiple tissues following administration, are promoted to engraft in animals consuming a HF, and mitigate hepatic oxidative stress in HF animals (Nyamandi et al., 2013). Inviting further exploration, we examined whether human-derived MSC administration would yield similar beneficial effects to the liver of HF animals. In the present study, we performed sequence-specific PCR to detect human transcripts from donor MSCs (Figure 4). Compared to our previously published work using flow cytometry-to quantitate the number of MSCs as they localize to various tissues (Nyamandi et al., 2013)_our present technique qualitatively identified MSCs by sequence-specific PCR, exclusive to the liver of HFM animals. These results confirmed that the transplanted MSCs successfully migrated to the liver and may therefore provide metabolic improvements to surrounding host tissue. Although this method enables detection of DNA variations between species, visualization via similar immunofluorescent staining as performed for our in vitro cell culture experiments would have been optimal. As mentioned previously, the liver was chosen because of its inherent ability to attract stem cells caused by lipid accumulation, its high concentration of mitochondria, and the presentation of metabolic inflammation following prolonged high-fat feeding (Ren et al., 2012; Zhao et al., 2015).
As a highly metabolically active tissue the liver contains a high concentration of mitochondria. The prolonged insult of metabolic inflammation induced by the HF phenotype has been shown to diminish healthy mitochondrial populations, resulting in decreased functional capacity of oxidative phosphorylation (OXPHOS) machinery (Satapati et al., 2015). Known to confer some of their benefits through the physical transfer of healthy donor mitochondria to the impaired host (Torralba et al., 2016), we examined the impact of MSC therapy to alter host mitochondrial function in the liver. As the most commonly dysfunctional enzyme complex of the electron transport system as observed in humans, complex I activity was examined using malate and glutamate as substrates (Mimaki et al., 2012). Animals treated with MSCs exhibited a greater maximal oxygen consumption rate (complex I) as well as elevated citrate synthase enzyme activity (Table 2 ). Collectively these results suggest that the improvements to metabolic function with MSCs may be due to increased mitochondria present within the host liver tissue. However, there is controversy since some research has suggested that while MSCs can rescue host mitochondrial function by transferring healthy mitochondria, cells with pathogenic mtDNA mutations do not undergo rescue (Cho et al., 2012). Contrarily, our results using an in vitro model of mitochondrial dysfunction show that MSC co-culture can alter mitochondrial morphology of cells from patients diagnosed with pathogenic mtDNA mutations (Table 1). 


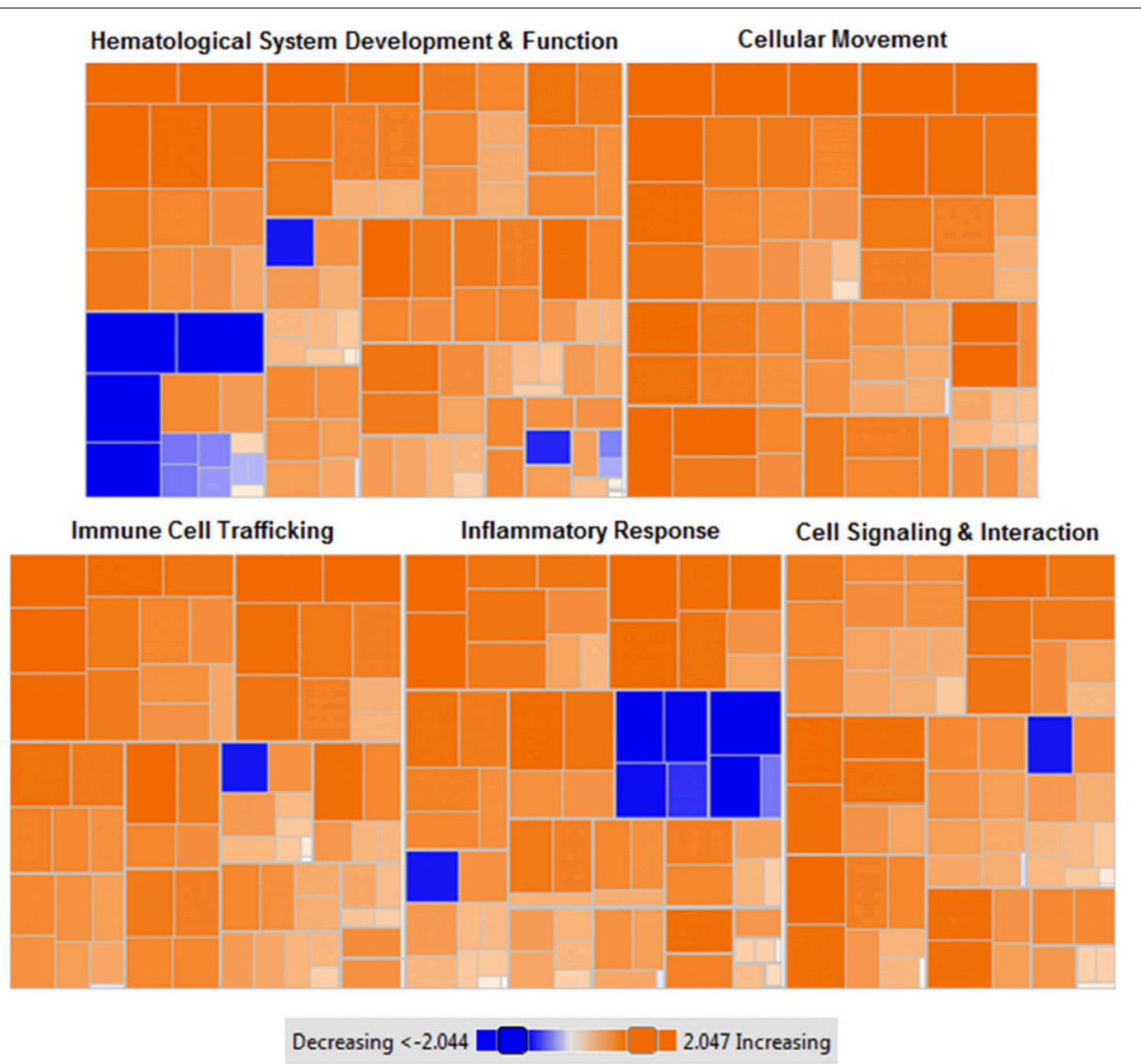

FIGURE 6 | Heat map of functional categories of closely related genes with differential gene expression following MSC administration. Enriched gene ontology terms of MSC treated liver tissues compared to control (saline) liver tissues using z-scores computed from microarray gene expression profiling. Orange: upregulated gene expression ( $z$-score $>0$ ), colorless: equal gene expression $(z$-score $=0$ ), blue: downregulated gene expression $(z$-score $<0)$. Data were generated from microarray gene expression data with each square corresponding to a single functional category. $n=7$ tissues per condition. $p<0.001$ for each (MSC treated vs. control).

Given that metabolic inflammation has been associated with an accumulation of increased mtDNA damage and impaired oxidative capacity (Yuzefovych et al., 2013; Arruda et al., 2014), the increase in citrate synthase activity following prolonged HF consumption indicates a potential increase to the viable mitochondrial population. As a by-product of healthy OXPHOS chemistry, ROS can positively impact surrounding tissues through improved cell signaling and transcriptional regulation, or negatively by oxidizing proteins and damaging DNA (Zorov et al., 2014). Since complexes I and III are the primary sites of ROS production (Brand, 2010), the collective increases in mitochondrial abundance and complex I mediated respiration in MSC treated animals may have resulted in elevated ROS production (Figure 5). The produced ROS is ultimately converted to $\mathrm{H}_{2} \mathrm{O}_{2}$ and sequestered by the glutathione and thioredoxin-2 (Trx2) antioxidant pathways (Stanley et al., 2011). Interestingly, recent research utilizing an in vivo model of ischemia-reperfusion injury has indicated that MSC therapy causes a decrease in Trx2 mRNA expression and that fibroblasts cultured in MSC conditioned media produce less ROS (Motegi et al., 2017). Since the ROS measurements in these experiments were collected over $2 \mathrm{~h}$, these data may provide insight to the pathway of ROS sequestration over a prolonged period. Contrarily, our measured elevations in ROS following MSC therapy were collected over minutes and were assayed using isolated mitochondria. These differences may demonstrate that although the total ROS generated are tempered by MSC therapy, snapshots in time using shorter experimental durations may capture some of the natural variability of ROS production under stressful respiratory activity (Aon et al., 2012). We next used a gene microarray to study global gene expression patterns between groups.

The most differentially expressed functional categories yielded several commonly increased functions including cell homing, cell migration, and cell recruitment (Figure 6, Table 4). Although counterintuitive, as MSCs are known to possess immunomodulatory properties, research investigating the manner in which MSCs migrate and home to tissues of interest has eluded to a similar mechanism as described for leukocyte homing (De Becker and Van Riet, 2016). This is supported by the significant upregulation of two acute phase proteins 
TABLE 4 | Functional categories of genes with differential expression in liver tissue following MSC therapy.

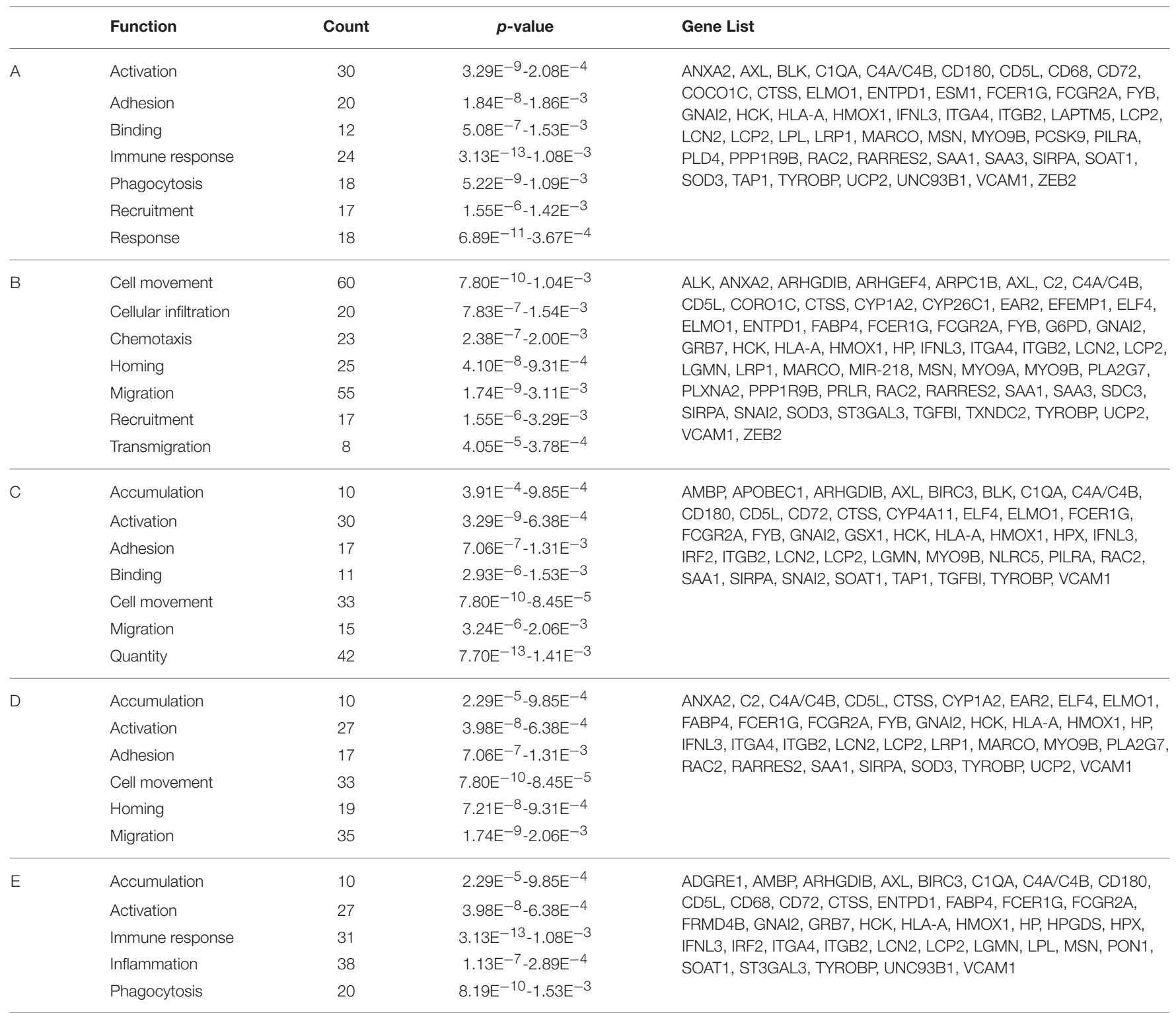

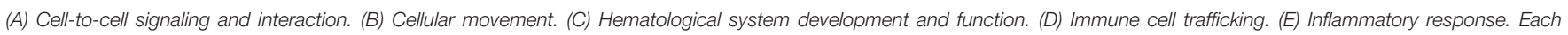

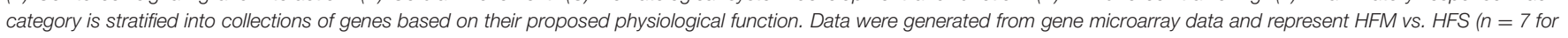
both groups).

produced by hepatocytes-serum amyloid A 1 (SAA1) and orosomucoid 1 (ORM1) which may act as hepatic signaling molecules following MSC therapy. Previously, SAA1 has been shown to be upregulated in bone-marrow and adipose derived MSCs and has been implicated in elevated energy-reserve and cholesterol metabolism (Liu et al., 2007). MSC lack expression of major histocompatibility complex (MHC) class II antigens (Le Blanc and Ringdén, 2005), and have been shown to inhibit B lymphocyte proliferation, chemotaxis, and differentiation (Corcione et al., 2005). This may enable MSCs to escape immune recognition by B lymphocytes while simultaneously promoting increased expression of genes responsible for cell homing, migration, and recruitment capacity (Chen et al., 2008).
Therefore, it is not surprising to identify that both hematopoietic and non-hematopoietic cell types are characterized by similar functional increases in cell homing and migration following MSC therapy. Further investigating the response to MSCs, we identified organ inflammation, quantity of leukocytes, and activation of B lymphocytes as the primary decreased functions (Figure 6). The prolactin receptor (PRLR) enables the action of prolactin on several sites throughout the body, namely the mammary glands, brain, and prostate (Sackmann-Sala et al., 2015). PRLR has, however, been identified on MSCs and other hematopoietic cell lineages and demonstrated capacity for MSC differentiation and B lymphocyte proliferation (Sackmann-Sala et al., 2015). Downregulation of PRLR may therefore enable 


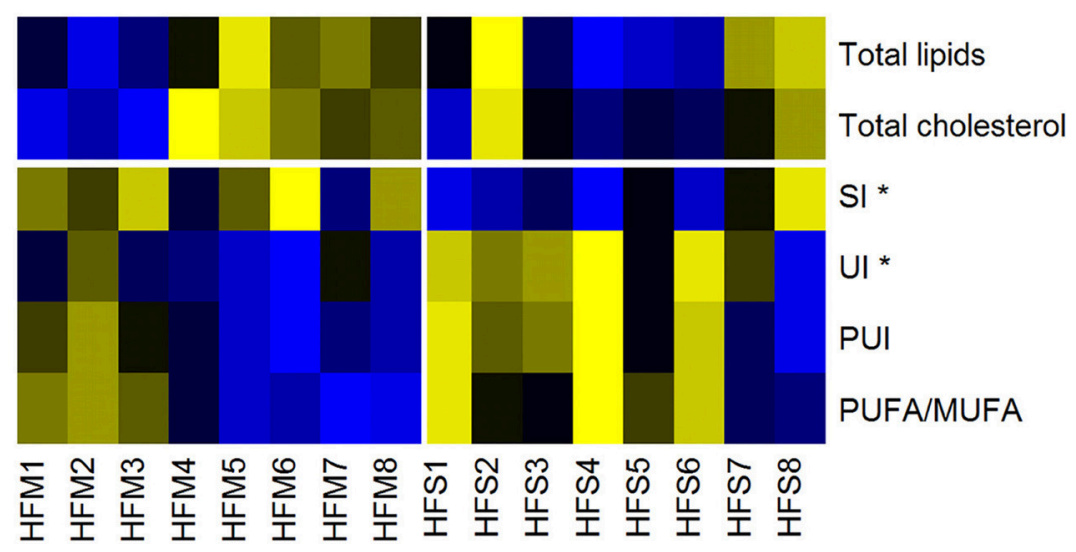

FIGURE 7 | Heat map of lipid liver metabolites/indices, measured by nuclear magnetic resonance. Yellow, High concentrations; Black, Medium concentrations; Blue, Low concentrations. SI, Saturation Index; UI, Unsaturation Index; PUI, Polyunsaturation Index; PUFA/MUFA, Polyunsaturated Fatty Acids/Monounsaturated Fatty Acids. High-fat fed animals were separated into two groups for analysis: MSC treated (HFM) and saline control (HFS) ${ }^{*}$ denotes statistical significance at $p<0.05$.

MSCs to migrate to their tissue of interest prior to differentiating. Previously shown to reduce systemic organ inflammation (Mei et al., 2010), suppress B lymphocyte differentiation (Corcione et al., 2005), and inhibit the formation of mixed lymphocyte cultures (Le Blanc et al., 2003), our data suggest that MSCs recapitulate this phenotype in a model of metabolic inflammation.

Further examination of the processes involved in cell migration with MSC treatment pinpointed actin cytoskeleton regulation as an upregulated key pathway. Actin cytoskeleton regulation has been implicated in modulating stem cell signaling and responsiveness (Müller et al., 2013). Through modifications of extracellular matrix composition, mechanical and molecular contact with stem cells can influence viability, and rate of engraftment (Battista et al., 2005). Although the exact role of actin cytoskeleton involvement was not examined in the present study, upregulation of this key pathway exclusive to HFM animals may demonstrate a host response to promote MSC viability and an elevated capacity for mitochondrial transfer via tunneling nanotubes (Islam et al., 2012). Furthermore, mitochondria are known to interact with cytoskeletal adaptor proteins (namely Miro1) for appropriate cellular distribution and regulation of mitochondrial morphology (Anesti and Scorrano, 2006; Ahmad et al., 2014). An upregulation of this pathway may therefore be in response to increases in mitochondrial abundance, as indicated by elevated citrate synthase activity and oxygen consumption rates following MSC therapy.

To provide a more comprehensive examination of the impact that MSC therapy had on the liver, we also explored metabolomic profiles. Metabolomics analysis revealed a significant increase in lipid saturation and a significant decrease in lipid unsaturation in the MSC treated group, while total lipid content remained constant (Figure 7, Table S2). This indicates a switch from polyunsaturated and monounsaturated fatty acids (PUFAs and MUFAs) to saturated fatty acids in animals treated with MSCs. Such a decrease in liver lipid unsaturation may be indicative of increased ROS, as the removal of oxidation-induced lipid peroxides by constitutive recycling of membrane phospholipids has been shown to reduce intracellular PUFA levels (Girón-Calle et al., 1997). Previous research investigating the role of PUFAs and MUFAs on oxidative metabolism using hepatic-derived HepG2 cells has shown that PUFAs enhance expression of ROS eliminating enzymes while MUFAs promote fatty acid oxidation and synthesis (Kohjima et al., 2009). These results indicate that variations in fatty acid composition may dictate ROS enzyme expression, ROS generation, and ROS sequestration. This coincides with our metabolic data in which we identified an increased level of ROS that was matched with increased mitochondrial abundance in the liver of MSC treated animals. Furthermore, the decreased PUFAs found in MSC treated livers may explain our elevated levels of ROS since the Trx 2 mediated antioxidant pathway has been shown to be downregulated in MSC treated animals whereas elevated MUFAs promote fatty acid oxidation and may generate excess ROS (Kohjima et al., 2009; Aon et al., 2012). In contrast, the water-soluble liver metabolites showed no significant difference between groups.

As a complex biological therapy, MSCs require exploration to identify the mechanisms of beneficial action. Our data indicate that MSC therapy rescues impaired mitochondrial morphology using an in vitro cell culture model, enhances host metabolic capacity, and induces widespread host gene shifting in an in vivo animal-model of metabolic inflammation. These data may provide insight into how MSCs exert some of their therapeutic impacts and help inform future clinical targets for improving host mitochondrial function.

\section{AUTHOR CONTRIBUTIONS}

JS, DH, TS, and AK designed and developed the research. CN, RS, MK, and JS conducted experiments, collected and analyzed data. $\mathrm{CN}$ and JS wrote the manuscript and all authors revised the manuscript critically for important intellectual content prior to final approval. All authors approved the final version of this manuscript, agree to be accountable for all aspects of the work in 
ensuring that questions related to the accuracy or integrity of any part of the work are appropriately investigated and resolved, and all persons designated as authors qualify for authorship, and all those who qualify for authorship are listed.

\section{ACKNOWLEDGMENTS}

This study was supported by NSERC and MitoCanada (JS). This research was supported by Ph.D. funding to $\mathrm{CN}$ from MitoCanada and Alberta Innovates-Health Solutions. We want to thank Virginia Berry for assistance in liver sample extraction, Dr. Nicole Prokopishyn for providing bone marrow-derived MSC and Elizabeth Newell for editing an earlier draft of this manuscript.

\section{SUPPLEMENTARY MATERIAL}

The Supplementary Material for this article can be found online at: https://www.frontiersin.org/articles/10.3389/fphys. 2018.01572/full\#supplementary-material

\section{REFERENCES}

Ahmad, T., Mukherjee, S., Pattnaik, B., Kumar, M., Singh, S., Rehman, R., et al. (2014). Miro1 regulates intercellular mitochondrial transport \& enhances mesenchymal stem cell rescue efficacy. EMBO J. 33, 994-1010. doi: $10.1002 / \mathrm{embj} .201386030$

Alán, L., Špaček, T., Pajuelo Reguera, D., Jaburek, M., and JeŽek, P. (2016). Mitochondrial nucleoid clusters protect newly synthesized mtDNA during doxorubicin- and ethidium bromide-induced mitochondrial stress. Toxicol. Appl. Pharmacol. 302, 31-40. doi: 10.1016/j.taap.2016. 04.011

Alcoser, S. Y., Kimmel, D. J., Borgel, S. D., Carter, J. P., Dougherty, K. M., and Hollingshead, M. G. (2011). Real-time PCR-based assay to quantify the relative amount of human and mouse tissue present in tumor xenografts. $B M C$ Biotechnol. 11:124. doi: 10.1186/1472-6750-11-124

Anderson, E. J., Lustig, M. E., Boyle, K. E., Woodlief, T. L., Kane, D. A., Lin, C-T., et al. (2009). Mitochondrial $\mathrm{H}_{2} \mathrm{O}_{2}$ emission and cellular redox state link excess fat intake to insulin resistance in both rodents and humans. J. Clin. Invest. 119, 573-581. doi: 10.1172/JCI37048

Anesti, V., and Scorrano, L. (2006). The relationship between mitochondrial shape and function and the cytoskeleton. Biochim. Biophys. Acta Bioenerg. 1757, 692-699. doi: 10.1016/j.bbabio.2006.04.013

Aon, M. A., Stanley, B. A., Sivakumaran, V., Kembro, J. M., O’Rourke, B., Paolocci, N., et al. (2012). Glutathione/thioredoxin systems modulate mitochondrial $\mathrm{H}_{2} \mathrm{O}_{2}$ emission: an experimental-computational study. J. Gen. Physiol. 139, 479-491. doi: 10.1085/jgp.201210772

Archer, S. L. (2013). Mitochondrial dynamics-mitochondrial fission and fusion in human diseases. N. Engl. J. Med. 369, 2236-2251. doi: 10.1056/NEJMra12 15233

Arruda, A. P., Pers, B. M., Parlakgül, G., Güney, E., Inouye, K., and Hotamisligil, G. S. (2014). Chronic enrichment of hepatic endoplasmic reticulummitochondria contact leads to mitochondrial dysfunction in obesity. Nat. Med. 20, 1427-1435. doi: 10.1038/nm.3735

Báez, A. L., Reynoso, M. N., Lo Presti, M. S., Bazán, P. C., Strauss, M., Miler, N., et al. (2015). Mitochondrial dysfunction in skeletal muscle during experimental Chagas disease. Exp. Mol. Pathol. 98, 467-475. doi: 10.1016/j.yexmp.2015.03.034

Battista, S., Guarnieri, D., Borselli, C., Zeppetelli, S., Borzacchiello, A., Mayol, L., et al. (2005). The effect of matrix composition of 3D constructs on embryonic stem cell differentiation. Biomaterials 26, 6194-6207. doi: 10.1016/j.biomaterials.2005.04.003
Table S1 | Primers for gene microarray validation using qRT-PCR and species specific qualitative PCR.

\section{Table S2 | P-values of liver metabolic indicies.}

Figure S1 | Mitochondrial morphology and mtDNA scoring classifications using a Zeiss LSM 700 confocal microscope and a 40x oil objective. Both fixed individual and live co -cultured skin fibroblast cells were manually classified using the following standardized images: A) 1 of 5 mitochondrial morphology categories and B) 1 of 2 categories for mtDNA structure. Mitochondrial morphology for skin fibroblasts collected using immunofluorescence against TOMM20.

Figure S2 | Typical trace of oxygen consumption following isolated mitochondria preparation using glutamate + malate as substrate, in liver tissue of C57BL/6 mice fed a high-fat diet. The blue line represents oxygen concentration within the chamber. The red line is the negative slope of the blue line (i.e., respiratory rate), normalized to tissue mass.

Video S1 | Bidirectional transfer of mitochondrial components between live fibroblasts co-cultured with MSCs. Video collected using confocal microscopy demonstrating bidirectional transfer of mitochondrial components between live fibroblasts co-cultured with MSCs. Images were collected following fluorescent labeling of fibroblasts with MitoTracker Deep Red (mitochondria, red) and PicoGreen (DNA, green). MSC's were separately fluorescently labeled with MitoTracker Red (mitochondria, teal) prior to co-culture.

Bernardo, M. E., and Fibbe, W. E. (2012). Safety and efficacy of mesenchymal stromal cell therapy in autoimmune disorders. Ann. N. Y. Acad. Sci. 1266, 107-117. doi: 10.1111/j.1749-6632.2012.06667.x

Brand, M. D. (2010). The sites and topology of mitochondrial superoxide production. Exp. Gerontol. 45, 466-472. doi: 10.1016/j.exger.2010.01.003

Chen, L., Tredget, E. E., Wu, P. Y. G., and Wu, Y. (2008). Paracrine factors of mesenchymal stem cells recruit macrophages and endothelial lineage cells and enhance wound healing. PLoS ONE 3:e1886. doi: 10.1371/journal.pone.0001886

Cho, Y. M., Kim, J. H., Kim, M., Park, S. J., Koh, S. H., Ahn, H. S., et al. (2012). Mesenchymal stem cells transfer mitochondria to the cells with virtually No mitochondrial function but not with pathogenic mtDNA mutations. PLoS ONE 7:e32778. doi: 10.1371/journal.pone.0032778

Colter, D. C., Class, R., DiGirolamo, C. M., and Prockop, D. J. (2000). Rapid expansion of recycling stem cells in cultures of plastic-adherent cells from human bone marrow. Proc. Natl. Acad. Sci.U.S.A. 97, 3213-3218. doi: $10.1073 /$ pnas.97.7.3213

Corcione, A., Benvenuto, F., Ferretti, E., Giunti, D., Cappiello, V., Cazzanti, F., et al. (2005). Human mesenchymal stem cells modulate B-cell functions. Blood 107, 367-372. doi: 10.1182/blood-2005-07-2657

De Becker, A., and Van Riet, I. (2016). Homing and migration of mesenchymal stromal cells: how to improve the efficacy of cell therapy? World J. Stem Cells 8 , 73-87. doi: $10.4252 /$ wjsc.v8.i3.73

Disatnik, M-H., Ferreira, J. C. B., Campos, J. C., Gomes, K. S., Dourado, P. M. M., Qi, X., et al. (2013). Acute inhibition of excessive mitochondrial fission after myocardial infarction prevents long-term cardiac dysfunction. J. Am. Heart Assoc. 2:e000461. doi: 10.1161/JAHA.113.000461

Fischer, T. D., Hylin, M. J., Zhao, J., Moore, A. N., Waxham, M. N., and Dash, P. K. (2016). Altered mitochondrial dynamics and TBI pathophysiology. Front. Syst. Neurosci. 10:29. doi: 10.3389/fnsys.2016.00029

Frezza, C., Cipolat, S., and Scorrano, L. (2007). Organelle isolation: functional mitochondria from mouse liver, muscle and cultured fibroblasts. Nat. Protoc. 2, 287-295. doi: 10.1038/nprot.2006.478

Gianni, P., Jan, K. J., Douglas, M. J., Stuart, P. M., and Tarnopolsky, M. A. (2004). Oxidative stress and the mitochondrial theory of aging in human skeletal muscle. Exp. Gerontol. 39, 1391-1400. doi: 10.1016/j.exger.2004.06.002

Giedt, R. J., Fumene Feruglio, P., Pathania, D., Yang, K. S., Kilcoyne, A., Vinegoni, C., et al. (2016). Computational imaging reveals mitochondrial morphology as a biomarker of cancer phenotype and drug response. Sci. Rep. 6:32985. doi: 10.1038/srep32985

Girón-Calle, J., Schmid, P. C., and Schmid, H. H. (1997). Effects of oxidative stress on glycerolipid acyl turnover in rat hepatocytes. Lipids 32, 917-23. 
Gregor, M. F., and Hotamisligil, G. S. (2011). Inflammatory mechanisms in obesity. Annu. Rev. Immunol. 29, 415-445. doi: 10.1146/annurev-immunol-031210-101322

Grohm, J., Kim, S-W., Mamrak, U., Tobaben, S., Cassidy-Stone, A., Nunnari, J., et al. (2012). Inhibition of Drp1 provides neuroprotection in vitro and in vivo. Cell Death Differ. 19, 1446-1458. doi: 10.1038/cdd.2012.18

Gronwald, W., Klein, M. S., Kaspar, H., Fagerer, S. R., Nürnberger, N., Dettmer, K., et al. (2008). Urinary metabolite quantification employing 2D NMR spectroscopy. Anal. Chem. 80, 9288-9297. doi: 10.1021/ac801627c

Guo, J., Jou, W., Gavrilova, O., and Hall, K. D. (2009). Persistent diet-induced obesity in male C57BL/6 mice resulting from temporary obesigenic diets. PLoS ONE 4:e5370. doi: 10.1371/journal.pone.0005370

Hittel, D. S., Axelson, M., Sarna, N., Shearer, J., Huffman, K. M., and Kraus, W. E. (2010). Myostatin decreases with aerobic exercise and associates with insulin resistance. Med. Sci. Sports Exerc. 42, 2023-2029. doi: 10.1249/MSS.0b013e3181e0b9a8

Hittel, D. S., Kraus, W. E., and Hoffman, E. P. (2003). Skeletal muscle dictates the fibrinolytic state after exercise training in overweight men with characteristics of metabolic syndrome. J. Physiol. 548, 401-410. doi: 10.1113/jphysiol.2002.036616

Hittel, D. S., Kraus, W. E., Tanner, C. J., Houmard, J. A., and Hoffman, E. P. (2005). Exercise training increases electron and substrate shuttling proteins in muscle of overweight men and women with the metabolic syndrome. J. Appl. Physiol. 98, 168-179. doi: 10.1152/japplphysiol.00331.2004

Hotamisligil, G. S. (2006). Inflammation and metabolic disorders. Nature 444, 860-867. doi: $10.1038 /$ nature 05485

Hsu, Y-C., Wu, Y-T., Yu, T-H., and Wei, Y-H. (2016). Mitochondria in mesenchymal stem cell biology and cell therapy: from cellular differentiation to mitochondrial transfer. Semin. Cell Dev. Biol. 52, 119-31. doi: 10.1016/j.semcdb.2016.02.011

Huang, D. W., Sherman, B. T., and Lempicki, R. A. (2009a). Bioinformatics enrichment tools: paths toward the comprehensive functional analysis of large gene lists. Nucleic Acids Res. 37, 1-13. doi: 10.1093/nar/gkn923

Huang, D. W., Sherman, B. T., and Lempicki, R. A. (2009b). Systematic and integrative analysis of large gene lists using DAVID bioinformatics resources. Nat. Protoc. 4, 44-57. doi: 10.1038/nprot.2008.211

Hubal, M. J., Reich, K. A., De Biase, A., Bilbie, C., Clarkson, P. M., Hoffman, E. P., et al. (2011). Transcriptional deficits in oxidative phosphorylation with statin myopathy. Muscle Nerve 44, 393-401. doi: 10.1002/mus.22081

Islam, M. N., Das, S. R., Emin, M. T., Wei, M., Sun, L., Westphalen, K., et al. (2012). Mitochondrial transfer from bone-marrow-derived stromal cells to pulmonary alveoli protects against acute lung injury. Nat. Med. 18, 759-765. doi: $10.1038 / \mathrm{nm} .2736$

Jheng, H. F., Tsai, P. J., Guo, S. M., Kuo, L. H., Chang, C. S. Su, I. J., et al. (2012). Mitochondrial fission contributes to mitochondrial dysfunction and insulin resistance in skeletal muscle. Mol. Cell. Biol. 32, 309-319. doi: 10.1128/MCB.05603-11

Kanehisa, M., Sato, Y., Kawashima, M., Furumichi, M., and Tanabe, M. (2016). KEGG as a reference resource for gene and protein annotation. Nucleic Acids Res. 44, D457-D462. doi: 10.1093/nar/gkv1070

Kim, K-Y., Perkins, G. A., Shim, M. S., Bushong, E., Alcasid, N., Ju, S., et al. (2015). DRP1 inhibition rescues retinal ganglion cells and their axons by preserving mitochondrial integrity in a mouse model of glaucoma. Cell Death Dis. 6:e1839. doi: 10.1038/cddis. 2015.180

Klein, M. S., Almstetter, M. F., Schlamberger, G., Nürnberger, N., Dettmer, K., Oefner, P. J., et al. (2010a). Nuclear magnetic resonance and mass spectrometrybased milk metabolomics in dairy cows during early and late lactation. J. Dairy Sci. 93, 1539-1550. doi: 10.3168/jds.2009-2563

Klein, M. S., Dorn, C., Saugspier, M., Hellerbrand, C., Oefner, P. J., and Gronwald, W. (2010b). Discrimination of steatosis and NASH in mice using nuclear magnetic resonance spectroscopy. Metabolomics 7, 237-246. doi: 10.1007/s11306-010-0243-6

Kohjima, M., Enjoji, M., Higuchi, N., Kato, M., Kotoh, K., Nakashima, M., et al. (2009). The effects of unsaturated fatty acids on lipid metabolism in HepG2 cells. Vitr. Cell. Dev. Biol. Anim. 45, 6-9. doi: 10.1007/s11626-0089144-7

Lane, M., Boczonadi, V., Bachtari, S., Gomez-Duran, A., Langer, T., Griffiths, A., et al. (2016). Mitochondrial dysfunction in liver failure requiring transplantation. J. Inherit. Metab. Dis. 39, 427-436. doi: 10.1007/s10545-016-9927-z

Le Blanc, K., and Ringdén, O. (2005). Immunobiology of human mesenchymal stem cells and future use in hematopoietic stem cell transplantation. Biol. Blood Marrow Transplant. 11, 321-334. doi: 10.1016/j.bbmt.2005.01.005

Le Blanc, K., Tammik, L., Sundberg, B., Haynesworth, S. E., and Ringden, O. (2003). Mesenchymal stem cells inhibit and stimulate mixed lymphocyte cultures and mitogenic responses independently of the major histocompatibility complex. Scand. J. Immunol. 57, 11-20. doi: 10.1046/j.1365-3083.2003.01176.x

Lee, R. K., Hittel, D. S., Nyamandi, V. Z., Kang, L., Soh, J., Sensen, C. W., et al. (2012). Unconventional microarray design reveals the response to obesity is largely tissue specific: analysis of common and divergent responses to dietinduced obesity in insulin-sensitive tissues. Appl. Physiol. Nutr. Metab. 37, 257-268. doi: 10.1139/h11-159

Liesa, M., Palacín, M., and Zorzano, A. (2009). Mitochondrial dynamics in mammalian health and disease. Physiol. Rev. 89, 799-845. doi: 10.1152/physrev.00030.2008

Liu, T. M., Martina, M., Hutmacher, D. W., Hui, J. H., Lee, E. H., and Lim, B. (2007). Identification of common pathways mediating differentiation of bone marrow- and adipose tissue-derived human mesenchymal stem cells into three mesenchymal lineages. Stem Cells 25, 750-760. doi: 10.1634/stemcells.2006-0394

Maijenburg, M. W., van der Schoot, C. E., and Voermans, C. (2012). Mesenchymal stromal cell migration: possibilities to improve cellular therapy. Stem Cells Dev. 21, 19-29. doi: 10.1089/scd.2011.0270

Mei, S. H. J., Haitsma, J. J., Dos Santos, C. C., Deng, Y., Lai, P. F. H., Slutsky, A. S., et al. (2010). Mesenchymal stem cells reduce inflammation while enhancing bacterial clearance and improving survival in sepsis. Am. J. Respir. Crit. Care Med. 182, 1047-1057. doi: 10.1164/rccm.201001-0010OC

Mimaki, M., Wang, X., McKenzie, M., Thorburn, D. R., and Ryan, M. T. (2012). Understanding mitochondrial complex I assembly in health and disease. Biochim. Biophys. Acta Bioenerg. 1817, 851-862. doi: $10.1016 /$ j.bbabio.2011.08.010

Motegi, S., Sekiguchi, A., Uchiyama, A., Uehara, A., Fujiwara, C., Yamazaki, S., et al. (2017). Protective effect of mesenchymal stem cells on the pressure ulcer formation by the regulation of oxidative and endoplasmic reticulum stress. Sci. Rep. 7:17186. doi: 10.1038/s41598-017-17630-5

Müller, P., Langenbach, A., Kaminski, A., and Rychly, J. (2013). Modulating the actin cytoskeleton affects mechanically induced signal transduction and differentiation in mesenchymal stem cells. PLOS ONE 8:e71283. doi: 10.1371 /journal.pone.0071283

Navarro, A., and Boveris, A. (2010). Brain mitochondrial dysfunction in aging, neurodegeneration and Parkinson's disease. Front. Aging Neurosci. 2:34. doi: 10.3389/fnagi.2010.00034

Nyamandi, V. Z., Johnsen, V. L., Hughey, C. C., Hittel, D. S., Khan, A., Newell, C., et al. (2013). Enhanced stem cell engraftment and modulation of hepatic reactive oxygen species production in diet-induced obesity. Obesity 22 , 721-729. doi: 10.1002/oby. 20580

Oh, J. Y., Lee, R. H., Yu, J. M., Ko, J. H., Lee, H. J., Ko, A. Y., et al. (2012). Intravenous mesenchymal stem cells prevented rejection of allogeneic corneal transplants by aborting the early inflammatory response. Mol. Ther. 20, 2143-2152. doi: $10.1038 / \mathrm{mt} .2012 .165$

Pieczenik, S. R., and Neustadt, J. (2007). Mitochondrial dysfunction and molecular pathways of disease. Exp. Mol. Pathol. 83, 84-92. doi: 10.1016/j.yexmp.2006.09.008

Reddy, P. H. (2014). Inhibitors of mitochondrial fission as a therapeutic strategy for diseases with oxidative stress and mitochondrial dysfunction. J. Alzheimers. Dis. 40, 245-256. doi: 10.3233/JAD-132060

Rego, A. C., Vesce, S., and Nicholls, D. G. (2001). The mechanism of mitochondrial membrane potential retention following release of cytochrome $\mathrm{c}$ in apoptotic GT1-7 neural cells. Cell Death Differ. 8, 995-1003. doi: 10.1038/sj.cdd.4400916

Ren, G., Chen, X., Dong, F., Li, W., Ren, X., Zhang, Y., et al. (2012). Concise review: mesenchymal stem cells and translational medicine: emerging issues. Stem Cells Transl. Med. 1, 51-58. doi: 10.5966/sctm.2011-0019

Rosca, M. G., and Hoppel, C. L. (2013). Mitochondrial dysfunction in heart failure Heart Fail. Rev. 18, 607-622. doi: 10.1007/s10741-012-9340-0

Sabouny, R., Fraunberger, E., Geoffrion, M., Ng, A. C-H., Baird, S. D. Screaton, R. A., et al. (2017). The Keap1-Nrf2 stress response pathway 
promotes mitochondrial hyperfusion through degradation of the mitochondrial fission protein Drp1. Antioxid. Redox Signal. 27, 1447-1459. doi: 10.1089/ars.2016.6855

Sackmann-Sala, L., Guidotti, J-E., and Goffin, V. (2015). Minireview: prolactin regulation of adult stem cells. Mol. Endocrinol. 29, 667-681. doi: 10.1210/me.2015-1022

Satapati, S., Kucejova, B., Duarte, J. A. G., Fletcher, J. A., Reynolds, L., Sunny, N. E., et al. (2015). Mitochondrial metabolism mediates oxidative stress and inflammation in fatty liver. J. Clin. Invest. 125, 4447-4462. doi: 10.1172/JCI82204

Sebastián, D., Palacín, M., and Zorzano, A. (2017). Mitochondrial Dynamics: coupling mitochondrial fitness with healthy aging. Trends Mol. Med. 23, 201-215. doi: 10.1016/j.molmed.2017.01.003

Sharp, W. W., Beiser, D. G., Fang, Y. H., Han, M., Piao, L., Varughese, J., et al. (2015). Inhibition of the mitochondrial fission protein dynamin-related protein 1 improves survival in a murine cardiac arrest model. Crit. Care Med. 43:e38-47. doi: 10.1097/CCM.0000000000000817

Shi, X. L., Gu, J. Y., Han, B., Xu, H. Y., Fang, L., and Ding, Y. T. (2010). Magnetically labeled mesenchymal stem cells after autologous transplantation into acutely injured liver. World J. Gastroenterol. 16, 3674-3679. doi: 10.3748/wjg.v16.i29.3674

Squillaro, T., Peluso, G., and Galderisi, U. (2016). Clinical trials with mesenchymal stem cells: an update. Cell Transplant. 25, 829-848. doi: 10.3727/096368915X689622

Stanley, B. A., Sivakumaran, V., Shi, S., McDonald, I., Lloyd, D., Watson, W. H., et al. (2011). Thioredoxin reductase-2 is essential for keeping low levels of $\mathrm{H}(2) \mathrm{O}(2)$ emission from isolated heart mitochondria. J. Biol. Chem. 286, 33669-33677. doi: 10.1074/jbc.M111.284612

Stephen, T-L., Higgs, N. F., Sheehan, D. F., Al Awabdh, S., Lopez-Domenech, G., Arancibia-Carcamo, I. L., et al. (2015). Miro1 regulates activity-driven positioning of mitochondria within astrocytic processes apposed to synapses to regulate intracellular calcium signaling. J. Neurosci. 35, 15996-16011. doi: 10.1523/JNEUROSCI.2068-15.2015

Sumida, M., Doi, K., Ogasawara, E., Yamashita, T., Hamasaki, Y., Kariya, T., et al. (2015). Regulation of mitochondrial dynamics by dynamin-related protein-1 in acute cardiorenal syndrome. J. Am. Soc. Nephrol. 26, 2378-2387. doi: 10.1681/ASN.2014080750

Thomas, A., Stevens, A. P., Klein, M. S., Hellerbrand, C., Dettmer, K., Gronwald, W., et al. (2012). Early changes in the liver-soluble proteome from mice fed a nonalcoholic steatohepatitis inducing diet. Proteomics 12, 1437-1451. doi: 10.1002/pmic.201100628

Torralba, D., Baixauli, F., and Sánchez-Madrid, F. (2016). Mitochondria know no boundaries: mechanisms and functions of intercellular mitochondrial transfer. Front. Cell Dev. Biol. 4:107. doi: 10.3389/fcell.2016.00107
Tweedie, C., Romestaing, C., Burelle, Y., Safdar, A., Tarnopolsky, M. A., Seadon, S., et al. (2011). Lower oxidative DNA damage despite greater ROS production in muscles from rats selectively bred for high running capacity. Am. J. Physiol. Regul. Integr. Comp. Physiol. 300, R544-R553. doi: 10.1152/ajpregu.002 50.2010

Wai, T., and Langer, T. (2016). Mitochondrial dynamics and metabolic regulation. Trends Endocrinol. Metab. 27, 105-117. doi: 10.1016/j.tem.2015.12.001

Wei, X., Yang, X., Han, Z., Qu, F., Shao, L., and Shi, Y. (2013). Mesenchymal stem cells: a new trend for cell therapy. Acta Pharmacol. Sin. 34, 747-754. doi: 10.1038/aps.2013.50

Wu, H., Southam, A. D., Hines, A., and Viant, M. R. (2008). High-throughput tissue extraction protocol for NMR- and MS-based metabolomics. Anal. Biochem. 372, 204-212. doi: 10.1016/j.ab.2007.10.002

Wu, Q., Xia, S-X., Li, Q-Q., Gao, Y., Shen, X., Ma, L., et al. (2016). Mitochondrial division inhibitor 1 (Mdivi-1) offers neuroprotection through diminishing cell death and improving functional outcome in a mouse model of traumatic brain injury. Brain Res. 1630, 134-143. doi: 10.1016/j.brainres.2015.11.016

Xu, F., Armstrong, R., Urrego, D., Qazzaz, M., Pehar, M., Armstrong, J. N., et al. (2016). The mitochondrial division inhibitor Mdivi-1 rescues mammalian neurons from anesthetic-induced cytotoxicity. Mol. Brain 9:35. doi: 10.1186/s13041-016-0210-x

Yuzefovych, L. V., Musiyenko, S. I., Wilson, G. L., and Rachek, L. I. (2013). Mitochondrial DNA damage and dysfunction, and oxidative stress are associated with endoplasmic reticulum stress, protein degradation and apoptosis in high fat diet-induced insulin resistance mice. PLOS ONE 8:e54059. doi: 10.1371/journal.pone.0054059

Zhao, L., Zhong, S., Qu, H., Xie, Y., Cao, Z., Li, Q., et al. (2015). Chronic inflammation aggravates metabolic disorders of hepatic fatty acids in high-fat diet-induced obese mice. Sci. Rep. 5:10222. doi: 10.1038/srep10222

Zorov, D. B., Juhaszova, M., and Sollott, S. J. (2014). Mitochondrial reactive oxygen species (ROS) and ROS-induced ROS release. Physiol. Rev. 94, 909-950. doi: 10.1152/physrev.00026.2013

Conflict of Interest Statement: The authors declare that the research was conducted in the absence of any commercial or financial relationships that could be construed as a potential conflict of interest.

Copyright (c) 2018 Newell, Sabouny, Hittel, Shutt, Khan, Klein and Shearer. This is an open-access article distributed under the terms of the Creative Commons Attribution License (CC BY). The use, distribution or reproduction in other forums is permitted, provided the original author(s) and the copyright owner(s) are credited and that the original publication in this journal is cited, in accordance with accepted academic practice. No use, distribution or reproduction is permitted which does not comply with these terms. 